\title{
Transient Receptor Potential Channels TRPM4 and TRPC3 Critically Contribute to Respiratory Motor Pattern Formation but not Rhythmogenesis in Rodent Brainstem Circuits
}

\author{
(1)Hidehiko Koizumi, ${ }^{1,}$ (1)Tibin T. John,, ${ }^{1,}$ (D) Justine X. Chia, ${ }^{1}$ Mohammad F. Tariq, ${ }^{1}$ Ryan S. Phillips, ${ }^{1,2}$ \\ Bryan Mosher, ${ }^{1}$ ○Yonghua Chen, ${ }^{1}$ Ryan Thompson, ${ }^{1}$ Ruli Zhang, ${ }^{1}$ ' Naohiro Koshiya, ${ }^{1}$ and ${ }^{\circledR}$ Jeffrey C. \\ Smith ${ }^{1}$
}

DOI:http://dx.doi.org/10.1523/ENEURO.0332-17.2018

${ }^{1}$ Cellular and Systems Neurobiology Section, National Institute of Neurological Disorders and Stroke, National Institutes of Health, Bethesda, MD 20892 and ${ }^{2}$ Department of Physics, University of New Hampshire, Durham, NH 03824

\begin{abstract}
Transient receptor potential channel, TRPM4, the putative molecular substrate for $\mathrm{Ca}^{2+}$-activated nonselective cation current $\left(I_{\text {CAN }}\right)$, is hypothesized to generate bursting activity of pre-Bötzinger complex (pre-BötC) inspiratory neurons and critically contribute to respiratory rhythmogenesis. Another TRP channel, TRPC3, which mediates $\mathrm{Na}^{+} / \mathrm{Ca}^{2+}$ fluxes, may be involved in regulating $\mathrm{Ca}^{2+}$-related signaling, including affecting TRPM4// ${ }_{\mathrm{CAN}}$ in respiratory pre-BötC neurons. However, TRPM4 and TRPC3 expression in pre-BötC inspiratory neurons and functional roles of these channels remain to be determined. By single-cell multiplex RT-PCR, we show mRNA expression for these channels in pre-BötC inspiratory neurons in rhythmically active medullary in vitro slices from neonatal rats and mice. Functional contributions were analyzed with pharmacological inhibitors of TRPM4 or TRPC3 in vitro as well as in mature rodent arterially perfused in situ brainstem-spinal cord preparations. Perturbations of respiratory circuit activity were also compared with those by a blocker of $I_{\text {CAN }}$. Pharmacologically attenuating endogenous activation of TRPM4, TRPC3, or $I_{\text {CAN }}$ in vitro similarly reduced the amplitude of inspiratory motoneuronal activity without significant perturbations of inspiratory frequency or variability of the rhythm. Amplitude perturbations were correlated with reduced inspiratory glutamatergic pre-BötC neuronal activity, monitored by multicellular dynamic calcium imaging in vitro. In more intact circuits in situ, the reduction of pre-BötC and motoneuronal inspiratory activity amplitude was accompanied by reduced post-inspiratory motoneuronal activity, without disruption of rhythm generation. We conclude that endogenously activated TRPM4, which likely mediates $I_{\text {CAN }}$, and TRPC3 channels in pre-BötC inspiratory neurons play fundamental roles in respiratory pattern formation but are not critically involved in respiratory rhythm generation.
\end{abstract}

Key words: Breathing; dynamic calcium imaging; I CAN; pre-Bötzinger complex

\section{Significance Statement}

Biophysical mechanisms generating the timing and patterning of rhythmic respiratory movements in mammals remain largely undefined. Calcium signaling-based theories for respiratory rhythm generation incorporating calcium-activated nonselective cation currents $\left(I_{\mathrm{CAN}}\right)$, postulated to be a type of transient receptor potential channel TRPM4, have been proposed but remain unproven. Here, we revealed that TRPM4 and TRPC3 channels are present and functionally active in rodent brainstem respiratory neurons, including in the inspiratory rhythm-generating circuits of the pre-Bötzinger complex. However, we established that these channels are not fundamentally involved in rhythm generation, but critically contribute to the formation of respiratory motor patterns. These results help resolve the longstanding debate in the field about the contributions of $I_{\text {CAN }}$ to rhythm and pattern generation in respiratory circuits. 


\section{Introduction}

Members of the transient receptor potential (TRP) channel superfamily, which mediate cationic current fluxes and control cell excitability and intracellular signaling, are involved in diverse aspects of brain function. Here we investigated roles of two subtypes of TRP channelsTRPM4 of the melastatin TRPM channel family and TRPC3 of the canonical TRPC channel family-in generating respiratory motor activity in the rodent brainstem-spinal cord. We established that these channels are expressed at the molecular level in populations of respiratory interneurons and motoneurons, they are endogenously activated during circuit activity, and they have a fundamental role in respiratory motor pattern generation.

Prominent, but unproven, $\mathrm{Ca}^{2+}$-based theories involving TRPM4 have been proposed for respiratory rhythm generation (Mironov, 2009; Del Negro et al., 2010) by excitatory neurons in the mammalian brainstem preBötzinger complex (pre-BötC), the established locus of interneurons in the medulla critical for inspiratory rhythm generation (Smith et al., 1991; Feldman and Del Negro, 2006). TRPM4, known to be a $\mathrm{Ca}^{2+}$-activated TRP channel (Guinamard et al., 2014), is postulated to be the molecular substrate of $\mathrm{Ca}^{2+}$-activated nonselective cation current $\left(I_{\text {CAN }}\right)$ in respiratory neurons (Crowder et al., 2007; Del Negro et al., 2010). TRPM4-mediated $I_{\text {CAN }}$ is proposed to be importantly involved in rhythm generation by functionally coupling activity-dependent intracellular $\mathrm{Ca}^{2+}$ signaling to neuronal depolarization and rhythmic neuronal activity generation (Del Negro et al., 2010; Guinamard et al., 2010). Previous studies (e.g., Koizumi and Smith, 2008) have shown that the rhythmically active neurons in pre-BötC circuits as well as neurons in downstream rhythmic drive transmission circuits exhibit large transient increases of intracellular $\mathrm{Ca}^{2+}$, potentially mediating TRPM4/ I CAN channel activity, during each respiratory cycle. TRPC3, on the other hand, is not directly activated by $\mathrm{Ca}^{2+}$, but mediates $\mathrm{Na}^{+} / \mathrm{Ca}^{2+}$ fluxes, and as a proposed $\mathrm{Ca}^{2+}$ store-operated channel, may be involved in regulating neuronal $\mathrm{Ca}^{2+}$-related signaling (Talavera et al., 2008;

Received September 22, 2017; accepted January 16, 2018; First published January 31,2018

The authors declare no competing financial interests.

Author contributions: H.K. and J.C.S. conceived of the study and designed the experiments; H.K., J.X.C., M.F.T., B.M., Y.C., and R.Z. performed the experiments; H.K., T.T.J., M.F.T., R.S.P., R.T., R.Z., and N.K. analyzed the data; H.K., T.T.J., M.F.T., N.K., and J.C.S. wrote the manuscript.

This research was supported by the Intramural Research Program of the $\mathrm{NIH}$, National Institute of Neurological Disorders and Stroke. RSP was supported by the Ted Giovanis Foundation through the NIH Office of Intramural Training and Education (OITE) and the NIH-UNH Graduate Partnership Program.

*H.K. and T.T.J. contributed equally to this work.

Correspondence should be addressed to either of the following: Hidehiko Koizumi, PhD, 49 Convent Dr, Room 2A22, NINDS, NIH, Bethesda, MD 20892. E-mail: koizumih@mail.nih.gov; or Jeffrey C. Smith, PhD, 49 Convent Dr, Room 2A10, NINDS, NIH, Bethesda, MD 20892. E-mail: smithj2@ninds.nih.gov.

DOI:http://dx.doi.org/10.1523/ENEURO.0332-17.2018

Copyright (C) 2018 Koizumi et al.

This is an open-access article distributed under the terms of the Creative Commons Attribution 4.0 International license, which permits unrestricted use, distribution and reproduction in any medium provided that the original work is properly attributed.
Birnbaumer, 2009; Guinamard et al., 2013), potentially affecting TRPM4// ${ }_{\mathrm{CAN}}$ in respiratory neurons. The role of TRPC3-mediated cationic currents $/ \mathrm{Ca}^{2+}$-related signaling in generating respiratory neuron activity has not been investigated.

TRPM4 and TRPC3 channels have been identified by mRNA/protein expression in tissue harvested from the mouse pre-BötC region (Crowder et al., 2007; BenMabrouk and Tryba, 2010), although they have not been demonstrated to be expressed in functionally identified respiratory neurons. Here we examined, by single-cell RTPCR, expression of TRPM4 and TRPC3 mRNA in functionally identified glutamatergic, glycinergic, and GABAergic inspiratory pre-BötC neurons as well as inspiratory motoneurons. We also examined neuronal channel expression by antibody labeling in the pre-BötC region and motor nuclei. We then investigated whether these channels contribute to respiratory circuit activity by pharmacological experiments with the selective channel inhibitors 9-phenanthrol for TRPM4 (Guinamard et al., 2014) and 3-pyrazole for TRPC3 (Kiyonaka et al., 2009), in both neonatal rat and mouse slice preparations with rhythmically active pre-BötC and respiratory motor circuits in vitro. Comparative analyses for these two species was important because there are numerous studies on respiratory rhythm and pattern generation using rats (e.g., Gray et al., 2001; Onimaru and Homma, 2003; Koizumi and Smith, 2008) or mice (e.g., Thoby-Brisson and Ramirez, 2001; Peña et al., 2004; Del Negro et al., 2005), so it is necessary to establish commonality of $\mathrm{Ca}^{2+}$-based mechanisms for respiratory rhythm and pattern generation. We also analyzed perturbations of pre-BötC excitatory circuit activity by dynamic $\mathrm{Ca}^{2+}$ imaging of inspiratory glutamatergic preBötC neurons with a genetically encoded $\mathrm{Ca}^{2+}$ sensor (Chen et al., 2013) in transgenic mice. We show that amplitudes of inspiratory pre-BötC neuronal activity, and the correlated amplitudes of motoneuronal output in vitro, are significantly reduced by TRPM4 and TRPC3 channel inhibitors. The pharmacological profile of inspiratory activity attenuation by inhibiting TRPM4 activation matched that with another proposed blocker of $I_{\mathrm{CAN}}$, flufenamic acid (FFA), consistent with the concept that TRPM4 mediates $I_{\text {CAN }}$ (Launay et al., 2002). In all cases, the attenuation of inspiratory circuit activity occurred without significant perturbations of the frequency of the inspiratory rhythm.

We also demonstrate critical involvement of TRPM4 and TRPC3 channels in regulating the amplitude of preBötC population activity and motor output patterns in more intact respiratory circuits active in arterially perfused brainstem-spinal cord in situ preparations from mature rats and mice. The reduction, by the channel inhibitors, of pre-BötC and motoneuronal inspiratory activity amplitude recorded electrophysiologically was accompanied by reductions of post-inspiratory motoneuronal activity. These amplitude perturbations also occurred without disrupting rhythm generation. In general, our results indicate that endogenous activation of these two types of TRP channels are involved in generating respiratory motor patterns, 
but critically not rhythm generation, in both neonatal and mature rodents.

\section{Materials and Methods}

\section{Animal procedures}

All animal procedures were approved by the Animal Care and Use Committee of the National Institute of Neurological Disorders and Stroke.

\section{Immunohistochemical labeling of TRPM4 and TRPC3 channels}

We examined fluorescence antibody labeling for TRPM4 and TRPC3 channels to identify channel expression in pre-BötC neurons in neonatal and mature rats and mice. In addition, we examined channel expression in relation to specific neurotransmitter phenotypes of neurons within the pre-BötC, BötC, and rostral ventral respiratory group (rVRG) regions. We used transgenic Cre-driver mouse strains crossed with Cre-dependent reporter transgenic strains to express fluorescent protein (tdTomato) in excitatory or inhibitory neurons by the cell type-specific promoters (Gong et al., 2007) vesicular glutamate transporter type-2 (VgluT2) or glycine transporter type-2 (GlyT2): VgluT2-tdTomato for glutamatergic neurons, and GlyT2-tdTomato for glycinergic neurons. The VgluT2-tdTomato strain was produced by crossing a VgluT2-ires-Cre strain (SIc17a6 ${ }^{\text {tm2(cre)Lowl } / J, ~ I M S R ~ J A X: ~}$ 016963, RRID: IMSR_JAX: 016963, Jackson Laboratory) with a Cre-dependent tdTomato reporter strain

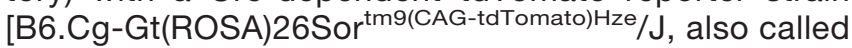
Ai9(RCL-tdT), IMSR JAX: 007909, RRID: IMSR_JAX: 007909, Jackson Laboratory]. The GlyT2-tdTomato mouse line was produced by crossing a GlyT2-Cre line [B6.FVB(cg)Tg(Slc6a5-cre)KF109Gsat/Mmucd, MMRRC 036055-UCD, RRID: MMRRC_036055-UCD, MMRRC, University of California, Davis] with the Ai9(RCL-tdT) line. In each of these double transgenic lines, we analyzed colabeling by TRPM4 or TRPC3 channel antibody in neurons prelabeled with tdTomato to identify expression of each channel in glutamatergic or glycinergic neurons.

The medulla oblongata from neonatal and mature rats or mice was fixed in $4 \%$ paraformaldehyde (wt/vol) in PBS, cryoprotected overnight at $4^{\circ} \mathrm{C}$ in $30 \%$ sucrose and $0.1 \mathrm{~m}$ PBS solution, and sectioned coronally ( 25 or $50 \mu \mathrm{m}$ ) with a freezing microtome. For fluorescent immunohistochemistry, floating sections were incubated with $10 \%$ donkey serum in PBS with Triton X-100 (0.3\%) and incubated for $48-72 \mathrm{~h}$ at room temperature with the following primary antibodies: polyclonal rabbit anti-TRPM4 (ab63080, Abcam ab63080, RRID: AB_956418, 1:1000) and polyclonal rabbit anti-TRPC3 (ACC-016, Alomone Labs, ACC016, RRID: AB_2040236, 1:200). We verified the specificity of these TRPM4 and TRPC3 antibodies by confirming the absence of immunoreactivity in the mouse medullary tissue sections with the primary antibody that was preincubated for $1 \mathrm{~h}$ at room temperature with saturating concentrations (10:1) of the antigenic blocking peptide (TRPM4: ab65597, Abcam, TRPC3: ACC-016, Alomone Labs). We also note that the specificity of the same TRPM4 and TRPC3 antibodies as those we used has been confirmed in a TRPM4 knockout mouse (Schattling et al., 2012) and a TRPC3 knockout mouse (Feng et al., 2013), respectively. Individual sections were then rinsed with PBS and incubated for $2 \mathrm{~h}$ with the secondary antibody (donkey anti-rabbit, Dylight 647). Individual sections were mounted on slides and covered with an anti-fading medium (Fluoro-Gel; Electron Microscopy Sciences). Fluorescent labeling of neurons was visualized with a laserscanning confocal imaging system (Zeiss LSM 510). Motoneurons were identified by antibody labeling for choline acetyltransferase (ChAT; goat anti-ChAT, Millipore AB144, RRID: AB_90650, 1:200; donkey anti-goat-Dylight $488,1: 500)$. TRP channel expression in cell bodies of interneurons was identified by the presence of channel immunoreactivity without ChAT antibody labeling. All images were color/contrast enhanced and adjusted with a thresholding filter in Adobe Photoshop.

For tallying the numbers of TRPM4 or TRPC3 channel antibody-labeled neurons in adult (3-5-mo-old) transgenic mice with glutamatergic or glycinergic neurons labeled with tdTomato fluorescent protein as presented in Results, we counted labeled neurons within a region (300-400 $\mu \mathrm{m}$ diameter depending on animal size, ventral to the nucleus ambiguus) in the coronal plane of $25-\mu \mathrm{m}$ thick tissue sections obtained from the pre-BötC, BötC, or rVRG regions on both sides of the medulla. The locations and rostro-caudal extent of each region were defined from our established anatomic criteria based on previous electrophysiological recording/cell activity mapping studies (e.g., Koizumi et al., 2016) as well as the present neuronal population activity recordings in the adult transgenic mouse in situ brainstem-spinal cord preparations. The anatomic criteria included the location, in the ventrolateral medullary reticular formation, of the BötC region at the levels of the compact division of nucleus ambiguus (NAc), the pre-BötC region at the levels of the semicompact division of NA (NAsc), and the rVRG region extending from near the level of obex to the caudal end of the pre-BötC. The rostro-caudal dimension of the pre-BötC region was $\sim 350 \mu \mathrm{m}$; the BötC region, $\sim 550 \mu \mathrm{m}$; and the rVRG region, $\sim 500 \mu \mathrm{m}$ in the 3-5-mo-old adult mice used for the analysis. We selected 4-6 of the coronal sections from each region so that sections at different levels clearly within the region were included for the bilateral cell counting, and our sample included sections from both the caudal and rostral half of each region to produce the regional tally of labeled neurons presented.

\section{Rhythmically active medullary slice preparations in vitro}

Rhythmically active transverse slices of the medulla oblongata that contained the pre-BötC and rostral end of the hypoglossal (XII) motor nucleus with intact XII nerve rootlets (Smith et al., 1991; Koizumi et al., 2013) were cut from neonatal [postnatal day 1-5 (P1-P5)] Sprague-Dawley rats of either sex (350-400- $\mu \mathrm{m}$-thick slices) or from neonatal (P3-P8) mice of either sex (300$400-\mu \mathrm{m}$-thick slices). The slice was superfused (4 ml/min) in vitro in a recording chamber $(0.2 \mathrm{ml})$ mounted on the stage of an upright laser scanning microscope with artifi- 
cial cerebrospinal fluid (ACSF) containing the following (in $\mathrm{mm}$ ): $124 \mathrm{NaCl}, 25 \mathrm{NaHCO}_{3}, 3 \mathrm{KCl}, 1.5 \mathrm{CaCl}_{2}, 1.0 \mathrm{MgSO}_{4}$, $0.5 \mathrm{NaH}_{2} \mathrm{PO}_{4}, 30 \mathrm{D}$-glucose equilibrated with $95 \% \mathrm{O}_{2}$ and $5 \% \mathrm{CO}_{2}\left(\mathrm{pH} 7.35-7.40\right.$ at $\left.27^{\circ} \mathrm{C}\right)$. During experiments, rhythmic respiratory network activity, monitored by recording inspiratory discharge in XII nerve rootlets (see below), was maintained by elevating the superfusate $\mathrm{K}^{+}$ concentration to $8-9 \mathrm{~mm}$.

\section{Calcium imaging and identification of pre-BötC respiratory neurons in vitro}

We employed $\mathrm{Ca}^{2+}$-sensors, either $\mathrm{Ca}^{2+}$-sensitive synthetic dye in slices from wild-type (WT) neonatal rats and mice or genetically encoded protein $\mathrm{Ca}^{2+}$ sensor with fast kinetics (GCaMP6f; Chen et al., 2013) in transgenic mice, to dynamically image $\mathrm{Ca}^{2+}$ activity of pre-BötC neurons for functional identification of respiratory neurons in vitro. In experiments with WT neonatal rats or mice, the $\mathrm{Ca}^{2+}$ imaging was combined with whole-cell patch-clamp recording from the identified rhythmically active inspiratory neurons. In some of these experiments, the neurons were retrogradely labeled through their contralaterally projecting axons using membrane semipermeable acetoxymethyl (AM) dye (Oregon Green BAPTA-1 AM: OGB, Invitrogen) microinjected in the midline region of the slice (Koshiya and Smith, 1999; Koizumi et al., 2013). The slice was incubated overnight (12 h) in ACSF containing antibiotics (500 units/l penicillin, 0.5 mg/l streptomycin, and 1 mg/l neomycin) to allow labeling of pre-BötC neurons and used for optical and electrophysiological recordings throughout the next day. In other experiments, we microinjected the dyes directly into the pre-BötC to label cells nonselectively regardless of their axonal projections (Koizumi et al., 2013). The microinjection pipette (tip size: 2-3 $\mu \mathrm{m}$ ) was placed at a depth of $150 \mu \mathrm{m}$ in the slice and $\sim 100 \mu \mathrm{m}$ away from the center of the pre-BötC to avoid excessive dye deposits and high background fluorescence in the imaging area of interest, and OGB was pressure injected ( $\sim 20$ psi, $2 \mathrm{~min})$. The slice was incubated $(>1 \mathrm{~h})$ for sufficient dye loading before the recording experiments.

In the set of imaging experiments with GCaMP6f in transgenic mice, we selectively expressed this $\mathrm{Ca}^{2+}$ sensor in glutamatergic neurons using Cre-driven expression controlled by the VgluT2 promoter (VgluT2-GCaMP6f mice). These mice were produced by crossing the VgluT2iris-Cre strain and a Cre-dependent GCaMP6f expressing

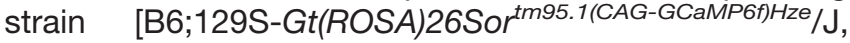
IMSR JAX: 024105, RRID: IMSR_JAX: 024105, Jackson Laboratory]. We imaged and quantified inspiratory-related GCaMP6f fluorescence transients in the pre-BötC glutamatergic neuronal population, which provides voltagedependent control of inspiratory frequency and functions as the critical rhythmogenic population (Koizumi et al., 2016).

In all experiments, optical imaging was performed with a Leica multiphoton laser scanning upright microscope (TCS SP5 II MP with DM6000 CFS system, LAS AF software), $20 \times$ water-immersion objective (N.A. 1.0), beamsplitter (560 nm), and emission filter (525/50, Semrock). A two-photon Ti:sapphire pulsed laser (MaiTai, Spectra
Physics) was used at 800-880 nm for $\mathrm{Ca}^{2+}$-sensitive dye or 910-920 nm for GCaMP6f, with DeepSee predispersion compensation. Dynamic $\mathrm{Ca}^{2+}$ fluorescence images (16 kHz bidirectional, 28 frames/s for $512 \times 512$-pixel scan) were acquired in real time along with electrophysiological signals of inspiratory XII nerve activities (LAS AF acquisition hardware and software electrophysiology module v.2.60). Simultaneous recording of these signals allowed us to functionally identify pre-BötC inspiratory neurons that were rhythmically active in phase with inspiratory network activity monitored by XII discharge. The infrared excitation laser for two-photon fluorescence was simultaneously used for transmission bright-field illumination to obtain a Dodt gradient contrast structural image to provide fluorescence and structural images matched to pixels. This structural imaging also allowed us to accurately place a patch pipette on functionally identified neurons in experiments using whole-cell recording. For experiments performed with GCaMP6f analyzing changes in dynamic fluorescence signals during application of pharmacological channel inhibitors (below), average fluorescence intensities $(F)$ of regions of interest were quantified for each frame, and dynamic fluorescence signals $(\Delta F)$ were represented as running baseline $\left(F_{0}\right)$-subtracted values $\left(F-F_{0}\right)$. We used $\Delta F$ not $\Delta F / F_{0}$ for the activity quantification because $\Delta F$ can be an order of magnitude brighter than $F_{0}$ at the laser wavelength optimal for GCaMP6f (910-920 nm), and $\Delta F$ was not proportional to $F_{0}$.

\section{Arterially perfused in situ brainstem-spinal cord preparations}

To investigate contributions of TRPM4/TRPC3 channels in generation of respiratory activity in more intact systems, we also performed experiments with in situ arterially perfused brainstem-spinal cord preparations from mature (3-4-wk-old) rats of either sex (Sprague-Dawley, 45-100 g) or adult (3-5-mo-old) mice of either sex (C57BL/6, 25-35 g; Paton, 1996; Smith et al., 2007). Preheparinized (1000 units, given intraperitoneally) rats/ mice were anaesthetized deeply with $5 \%$ isoflurane until loss of the paw withdrawal reflex, and the portion of the body caudal to the diaphragm was removed. The head and thorax were immersed in ice-chilled carbogenated ACSF solution containing the following (in $\mathrm{mm}$ ): 1.25 $\mathrm{MgSO}_{4}, 1.25 \mathrm{KH}_{2} \mathrm{PO}_{4}, 5.0 \mathrm{KCl}, 25 \mathrm{NaHCO}_{3}, 125 \mathrm{NaCl}, 2.5$ $\mathrm{CaCl}_{2}, 10$ dextrose, and 0.1785 polyethylene glycol. The brain was decerebrated at a precollicular level, and the descending aorta, thoracic phrenic nerve (PN), and cervical vagus nerves (VN) were surgically isolated. The dorsal brainstem was exposed by craniotomy and cerebellectomy. The preparation was transferred to a recording chamber and secured in a stereotaxic head frame with the dorsal side up. The descending aorta was cannulated with a double lumen catheter (DLR-4, Braintree Scientific) for ACSF perfusion with a peristaltic roller pump (505D, Watson-Marlow) and for recording of perfusion pressure with a pressure transducer. The ACSF perfusate was gassed with $95 \% \mathrm{O}_{2}-5 \% \quad \mathrm{CO}_{2}$ and maintained at $31^{\circ} \mathrm{C}$. Vecuronium bromide or rocuronium bromide $(2-4 \mu \mathrm{g} / \mathrm{ml}$; 
SUN Pharmaceutical Industries) was added to the perfusate to block neuromuscular transmission. Vasopressin (200-400 pM as required; APP Pharmaceuticals) was added to the perfusate to raise and maintain perfusion pressure between 70 and $80 \mathrm{mmHg}$ (Pickering and Paton, 2006).

\section{Electrophysiological recording in vitro and in situ}

To monitor inspiratory network activity and motor output in the rhythmically active neonatal rat and mouse medullary slice preparations in vitro, XII motoneuron population activity was recorded from XII nerve rootlets with fire-polished glass suction electrodes (50-100- $\mu \mathrm{m}$ inner diameter). Extracellular recordings of inspiratory and post-inspiratory motoneuronal activity from VN, and inspiratory activity from $\mathrm{PN}$, in the in situ perfused mature rat and mouse preparations were also obtained with suction electrodes (150-200- $\mu \mathrm{m}$ inner diameter). Signals in all cases were amplified (50,000-100,000×, CyberAmp 380, Molecular Devices), bandpass filtered $(0.3-2 \mathrm{kHz})$, digitized $(10 \mathrm{kHz})$ with an $\mathrm{AD}$ converter [Power Lab, AD Instruments or Cambridge Electronics Design] and then rectified and integrated digitally with Chart software (AD Instruments) for the in vitro slice preparations or Spike 2 software (Cambridge Electronics Design) for the perfused in situ preparations. Extracellular population activity from the pre-BötC in the perfused in situ preparations was also recorded by a dorsal approach with a fine-tipped glass pipette (3-5-M $\Omega$ resistance) filled with $0.5 \mathrm{~m}$ sodium acetate (Sigma-Aldrich), or in some cases with a tungsten microelectrode (3-4 M $\Omega$ ), which was positioned by a computer-controlled 3D micromanipulator (MC2000, Märzhäuser). The precise location of the pre-BötC was determined by the anatomic coordinates that we initially defined by mapping neuronal activity of medullary respiratory neurons within the ventral respiratory column. The pre-BötC is readily identified by a characteristic pattern of pre-inspiratory/inspiratory population activity and is distinguishable from the more rostral BötC region, which has a characteristic profile of post-inspiratory and augmenting expiratory activities, and from the more caudal rVRG region, which has an established profile of augmenting inspiratory population activity.

For the in vitro slice experiments in which cytoplasm was harvested from rhythmically active pre-BötC neurons for molecular analyses (below), whole-cell current-clamp recordings were first obtained with a HEKA EPC-9 patchclamp amplifier (HEKA Electronics) controlled by PatchMaster software (HEKA; 2.9-kHz low-pass filter, sampled at $100 \mathrm{kHz}$ ). Recording electrodes (borosilicate glass pipette, 4-6 M $\Omega$ ), positioned with microdrives (Scientifica), contained (in mm): $130.0 \mathrm{~K}$-gluconate, $5.0 \mathrm{Na}$-gluconate, 3.0 NaCl, 10.0 Hepes, 4.0 Mg-ATP, 0.3 Na-GTP, and 4.0 sodium phosphocreatine, $\mathrm{pH} 7.3$ using $\mathrm{KOH}$.

\section{Single-cell multiplex RT-PCR for mRNA expression profiling in functionally identified pre-BötC respiratory neurons}

After whole-cell recording, cytoplasm of the imaged and electrophysiologically identified pre-BötC respiratory neurons was aspirated as completely as possible into the patch pipette under visual control and then immediately expelled into a thin-walled tube for PCR containing reverse-transcription reagents (Invitrogen; Koizumi et al., 2013). To avoid contamination, we continuously applied positive pressure to the autoclaved glass pipettes used for whole-cell recording as the pipette was advanced in the slice to the targeted neuron. Single-cell multiplex RT-PCR (scmRT-PCR) was subsequently performed on the cytoplasm to probe for mRNA for TRPM4 and TRPC3 channels, VgluT2, GlyT2, and glutamic acid decarboxylase 67 (GAD67) to identify channel expression in pre-BötC inspiratory glutamatergic and glycinergic/GABAergic neurons. First-strand CDNA was synthesized for $1.5 \mathrm{~h}$ at $50^{\circ} \mathrm{C}$ in a mixture of $\mathrm{MgCl}_{2}(2 \mu \mathrm{l}, 25 \mathrm{~mm})$, dNTPs $(1 \mu \mathrm{l}, 10 \mathrm{~mm})$, BSA $(0.7 \mu \mathrm{l}, 143 \mathrm{ng} / \mu \mathrm{l})$, random hexamers $(1 \mu \mathrm{l}, 50 \mathrm{ng} / \mu \mathrm{l})$, oligodT (0.7 $\mu \mathrm{l}, 0.5 \mu \mathrm{g} / \mu \mathrm{l})$, RNaseOUT (1.2 $\mu \mathrm{l}$, 40 units $/ \mu \mathrm{l})$, DTT (1 $\mu \mathrm{l}$, $0.1 \mathrm{M})$, and SuperScriptIII RT (1 $\mu \mathrm{l}, 200$ units/ $\mu \mathrm{l})$. The entire reaction was either immediately used as template for multiplex PCR or frozen at $-80^{\circ} \mathrm{C}$ until assayed. After reverse transcription, the cDNA for each target mRNA was amplified simultaneously in a multiplex PCR procedure, using primers for TRPM4 (forward, 5'-AAGCTCCCCTGCGCCATCGT-3'; reverse, 5'-AGGGCAGGCCGCGAATGGAA), TRPC3 (forward, 5'-TGGGTTCTCGGGATGATGTGGT-3'; reverse, 5'GGGACCAGACTGAAGGGTGGAGG), VgluT2 (forward, 5'TGTTCTGGCTTCTGGTGTCTTACGAGAG-3'; reverse, 5'TTCCCGACAGCGTGCCAACA-3'), GlyT2 (forward, 5'-TCT GCATGACTGCCTATCCG-3'; reverse, 5'-AACACAGGCTT GTGTGTGCG-3'), and GAD67 (forward, 5'-ACCCTGGTGCCCGCTTCC-3'; reverse, 5'-TATTGGTATTGGCAGTTGAT GTC-3'). The first multiplex PCR was performed as a hotstart reaction in a final volume of $50 \mu \mathrm{l}$ containing $12 \mu \mathrm{l}$ reverse transcription reaction, $20-50 \mathrm{pM}$ of each primer, 0.2 mM dNTPs, and 10× High Fidelity PCR buffer with $2 \mathrm{~mm}$ $\mathrm{MgCl}_{2}$ and $5 \mathrm{U}$ Platinum Taq High Fidelity DNA polymerase (Invitrogen). The reaction mixtures were heated to $94^{\circ} \mathrm{C}$ for 2 min, and 30 cycles $\left(94^{\circ} \mathrm{C}, 30 \mathrm{~s} ; 55^{\circ} \mathrm{C}, 30 \mathrm{~s} ; 68^{\circ} \mathrm{C}, 1 \mathrm{~min}\right)$ of PCR were followed by a final elongation period of $10 \mathrm{~min}$ at $68^{\circ} \mathrm{C}$. The second round of PCR amplification was performed as individual reactions with primers for TRPM4 (forward, 5'-GGCCCAAGATTGTCATCGTG-3'; reverse, 5'TTGGCATACTGGGACACACA-3'), TRPC3 (forward, 5'CTGCAAGCCACCAAAGCGCAC-3'; reverse, 5'-CATGTTG AGCAGAACGACCACCA-3'), VgluT2 (forward, 5'-AGGTACATAGAAGAGAGCATCGGGGAGA-3'; reverse, 5'-CAC TGTAGTTGTTGAAAGAATTTGCTTGCTC-3'), GlyT2 (forward, 5'-TCTGCATGACTGCCTATCCG-3'; reverse, 5'-CAT GGTGTCAAGTCCAAGCG-3'), and GAD67 (forward, 5'GGACTTCCACCACCCACAC-3'; reverse, 5'-CTAAACCA ATGATATCCAAACCAG-3'), using $1 \mu \mathrm{l}$ of the first PCR reaction product under similar conditions with the following modifications: $50 \mathrm{pM}$ of each primer pair and 25 thermal cycles. Aliquots $(10 \mu \mathrm{l})$ of PCR products were separated and visualized in a SYBR Green-stained agarose gel $(2 \%)$ by electrophoresis. The expected sizes of PCR-generated fragments were: TRPM4 (301 bp), TRPC3 (522 bp), VgluT2 (315 bp), GlyT2 (701 bp), and GAD67 (185 bp). To ensure that the PCR signal arose from the cytoplasm of the recorded cell, the same RT-PCR assays were run on pipette solution collected from negative control "mock harvests" in each exper- 
iment (Koizumi et al., 2013). These assays were performed on the pipette solution after the pipette was advanced into the slice and withdrawn without extracting cell contents. In all scmRT-PCR assays, $100 \mathrm{pg}$ total rat brain RNA (Ambion) was also run as RT template to serve as a positive control.

\section{Analyses of TRP channel contributions to respiratory rhythm and motor pattern generation in vitro and in situ}

We performed combined electrophysiological and pharmacological experiments to probe for the contributions of endogenously active TRPM4 and TRPC3 channels to respiratory rhythm and motor pattern generation in the in vitro slice preparations. We analyzed the time course of perturbations of the inspiratory burst frequency, amplitude, and duration of integrated XII inspiratory motor output after application to the slice bathing solution of the putative selective TRPM4 channel inhibitor (9-phenanthrol, Millipore, 10-50 $\mu \mathrm{m}$; Guinamard et al., 2014), the selective TRPC3 channel inhibitor (Pyrazole compound-3: Pyr3, Millipore, 10-50 $\mu \mathrm{M}$; Kiyonaka et al., 2009), and for comparison, the putative $I_{\text {CAN }}$ blocker flufenamic acid (FFA, Sigma-Aldrich, 20-75 $\mu \mathrm{m}$; Teulon, 2000; Guinamard et al., 2013). In experiments with mouse slices expressing GCaMP6f in glutamatergic neurons, the dynamic $\mathrm{Ca}^{2+}$ activity within the pre-BötC (regional and single-neuron $\Delta F$ ) was measured for 2.5-min intervals before and starting at $5,10,20$, and $30 \mathrm{~min}$ after drug application to analyze local perturbations of neuronal activity within the pre-BötC rhythm-generating circuit accompanying perturbations of XII activity, which was continuously recorded throughout the experiments. Mean peak $\Delta F$ values computed for the 2.5-min periods were normalized to the mean peak $\Delta F$ values during control (before drug application) 2.5-min periods.

Contributions of TRPM4 and TRPC3 channels to respiratory rhythm and motor pattern generation in the mature rat and mouse arterially perfused brainstem-spinal cord preparations in situ were also analyzed by adding 9-phenanthrol $(20-50 \mu \mathrm{M})$ and Pyr3 $(50 \mu \mathrm{M})$ to the perfusion solution. Perturbations of the inspiratory PN motor output as well as $\mathrm{VN}$ inspiratory and post-inspiratory activity were analyzed. Throughout these experiments, the perfusion pressure was maintained with vasopressin added to the perfusion solution as required or by adjusting the perfusion pump speed to avoid possible effects of perfusion pressure changes on respiratory activity, since 9-phenanthrol and Pyr3 caused reductions $(10-20 \mathrm{mmHg})$ in perfusion pressure, consistent with the proposed role of TRPM4 and TRPC 3 channels in the control of vascular smooth muscle tone (Brayden et al., 2008).

\section{Signal analyses of respiratory parameters}

All digitized electrophysiological signals were analyzed by automated procedures to extract respiratory parameters from integrated nerve or neuronal population activities, performed with IDL (Exelis VIS) and Matlab (R2016a, Matlab, RRID: SCR_001622) software using the NIH high-performance computing Biowulf cluster. Inspiratory events were detected from the smoothed integrated XII (in vitro) or PN (in situ) signals via a $300-\mathrm{ms}$ window moving average and peak detection algorithm that calculated a threshold-based zero derivative (positive peak) point. After peak detection, inspiratory activity time $\left(T_{1}\right)$, expiratory interval time $\left(T_{\mathrm{E}}\right)$, respiratory period $\left(T_{\mathrm{TOT}}\right)$, and frequency $\left(f_{\mathrm{R}}=1 / T_{\text {ТОт }}\right)$ were computed. $T_{1}$ was measured as the original integrated burst width at $20 \%$ of the peak height above baseline; $T_{\mathrm{E}}$ was calculated as $T_{\mathrm{TOT}}-T_{1}$. Inspiratory amplitude was calculated by subtracting the local baseline value from the peak value of the integrated signals. The endpoint of the parameter quantification was defined when inspiratory amplitude declined to either a quasisteady state value as assessed by inspection, or to noise level with the disappearance of inspiratory activity. Representative time courses of these parameters were extracted by a 300-s-window, time-based moving median. Data were then pooled per experimental condition, and summary time courses were computed with the parameter values normalized to mean values during the control period (300-0 s before drug application).

We also quantified effects of the pharmacological manipulations on the regularity of the inspiratory rhythm by analyzing Poincaré plots of periods for 80 inspiratory bursts before and after drug application as the integrated inspiratory amplitude reached the defined endpoint. Short- and long-term period variability was quantified by plotting each $T_{\text {TОт }}$ as a function of the preceding $T_{\text {ТОт }}$ and fitting a Gaussian distribution to these points projected onto the line perpendicular to the $y=x$ line (with standard deviation SD1) and the points projected onto the $y=x$ line (with standard deviation SD2). SD1 represents total burstto-burst period variability, and SD2 represents total variability minus burst-to-burst variability, serving as measurements of short- and long-term rhythm regularity, respectively (Tulppo et al., 1996; Fishman et al., 2012). Values of SD1 and SD2 during periods of drug application were normalized to control values. Coefficients of variation of inspiratory burst periods were also calculated to measure mean normalized variability of $T_{\text {TOT }}$ over the same time intervals used to determine SD1 and SD2.

For the in situ experiments, in addition to quantifying the respiratory parameters indicated above $\left(T_{\mathrm{TOT}}, T_{\mathrm{I}}, T_{\mathrm{E}}\right.$, $f_{\mathrm{R}}$ ), we analyzed perturbations of amplitudes and durations of individual phases of the respiratory cycle. Amplitudes and durations of inspiratory activity in PN and VN recordings and post-inspiratory activity in $\mathrm{VN}$ recordings were analyzed from integrated, cycle phase-triggered neurograms aligned at the onset of inspiration defined by PN activity. Successive cycle-triggered traces were either overlaid (PN and VN separately) or represented as a dynamic raster plot to depict temporal profiles of activity before, during, and after drug application periods.

For statistical analysis (Table 1), the endpoint values, as described above, of each experiment within a group were compared with the control values with a two-sided Wilcoxon signed-rank test. Correlation analyses on data from imaging experiments were performed by computing either Pearson's $(r)$ or Spearman's rank $\left(r_{s}\right)$ correlation coefficient. In all tests, significance level was set at $p<0.05$. 
Table 1. Summary of statistics from figures

\begin{tabular}{|c|c|c|c|}
\hline Figure & Parameter & Type of test & $p$-value \\
\hline $4 A$ & 9-Phen: XII Amp, $f_{R}, T_{1}$ & Two-sided Wilcoxon signed rank & $0.031,0.31,0.031$ \\
\hline $4 B$ & Pyr3: XII Amp, $\mathrm{f}_{\mathrm{R}}, \mathrm{T}_{\mathrm{I}}$ & Two-sided Wilcoxon signed rank & $0.031,0.85,0.062$ \\
\hline $4 C$ & FFA: XII Amp, $f_{R}, T_{1}$ & Two-sided Wilcoxon signed rank & $0.031,1.00,0.031$ \\
\hline $5 A$ & 9-Phen: XII Amp, $f_{R}, T_{1}$ & Two-sided Wilcoxon signed rank & $0.031,0.44,0.031$ \\
\hline $5 B$ & Pyr3: XII Amp, $f_{R}, T_{I}$ & Two-sided Wilcoxon signed rank & $0.031,0.16,0.031$ \\
\hline $5 C$ & FFA: XII Amp, $f_{R}, T_{1}$ & Two-sided Wilcoxon signed rank & $0.031,0.31,0.062$ \\
\hline \multirow[t]{3}{*}{$6 B$} & 9-Phen: SD1, SD2 & Two-sided Wilcoxon signed rank & $0.31,0.69$ \\
\hline & Pyr3: SD1, SD2 & Two-sided Wilcoxon signed rank & $1.00,0.44$ \\
\hline & FFA: SD1, SD2 & Two-sided Wilcoxon signed rank & $0.31,0.56$ \\
\hline \multirow[t]{3}{*}{$6 D$} & 9-Phen: SD1, SD2 & Two-sided Wilcoxon signed rank & $0.094,0.094$ \\
\hline & Pyr3: SD1, SD2 & Two-sided Wilcoxon signed rank & $0.84,0.84$ \\
\hline & FFA: SD1, SD2 & Two-sided Wilcoxon signed rank & $0.56,0.56$ \\
\hline $8 A$ & Control: $\mathrm{f}_{\mathrm{R}}, \mathrm{XII}$ Amp, $\Delta \mathrm{F}$ & Spearman's rank correlation & $0.70,0.74,0.62$ \\
\hline \multirow[t]{3}{*}{$8 B$} & 9-Phen: $\mathrm{f}_{\mathrm{R}}, \mathrm{XII}$ Amp, $\Delta \mathrm{F}$ & Spearman's rank correlation & $0.52,0.00000048,0.017$ \\
\hline & Pyr3: $\mathrm{f}_{\mathrm{R}}, \mathrm{XII}$ Amp, $\Delta \mathrm{F}$ & Spearman's rank correlation & $0.60,0.0011,0.0028$ \\
\hline & FFA: $f_{R}, X I I A m p, \Delta F$ & Spearman's rank correlation & $0.22,0.00070,0.031$ \\
\hline \multirow[t]{3}{*}{$8 C$} & 9-Phen: XII Amp vs $\Delta \mathrm{F}$ & Pearson's linear correlation & 0.000032 \\
\hline & Pyr3: XII Amp vs $\Delta \mathrm{F}$ & Pearson's linear correlation & 0.0000052 \\
\hline & FFA: XII Amp vs $\Delta \mathrm{F}$ & Pearson's linear correlation & 0.0013 \\
\hline \multirow[t]{2}{*}{$12 A$} & 9-Phen: pre-BötC, VN, $P N, f_{R}, T_{1}$ & Two-sided Wilcoxon signed rank & $\begin{array}{l}0.031,0.031,0.031 \\
0.031,0.44\end{array}$ \\
\hline & Pyr3: pre-BötC, VN, PN, $f_{R}, T_{1}$ & Two-sided Wilcoxon signed rank & $\begin{array}{l}0.0078,0.0078,0.016 \\
0.016,0.25\end{array}$ \\
\hline \multirow[t]{2}{*}{$12 B$} & 9-Phen: pre-BötC, VN, $P N, f_{R}, T_{1}$ & Two-sided Wilcoxon signed rank & $\begin{array}{l}0.031,0.016,0.016 \\
0.031,0.031\end{array}$ \\
\hline & Pyr3: pre-BötC, VN, PN, $\mathrm{f}_{\mathrm{R}}, \mathrm{T}_{\mathrm{I}}$ & Two-sided Wilcoxon signed rank & $\begin{array}{l}0.031,0.031,0.031 \\
0.44,0.031\end{array}$ \\
\hline
\end{tabular}

\section{Results}

Immunohistochemical labeling of TRPM4 and TRPC3 channels in pre-BötC neurons, regions of the ventral respiratory column adjacent to the pre-BötC, and motoneurons

TRPM4 and TRPC3 channel antibodies labeled neurons bilaterally within the pre-BötC region (Fig. 1) in medullary slices from neonatal and mature rats/mice ( $n=3$ each). These channels were also labeled by antibody in (1) motoneurons defined by ChAT immunolabeling within nucleus ambiguus (NA) and the XII motor nucleus containing subpopulations of respiratory motoneurons; (2) neurons within the medullary reticular formation zone dorsal to pre-BötC where inspiratory XII premotor neurons are distributed (Koizumi et al., 2008; Revill et al., 2015); (3) neurons within the rostral ventral respiratory group (rVRG) region, adjacent and caudal to the pre-BötC, where bulbospinal respiratory neurons are localized; and (4) neurons in the BötC region containing respiratory neurons rostral to the pre-BötC. TRPM4 and TRPC3 channels are not exclusively expressed in these regions, but as indicated by antibody labeling are widely expressed in neurons throughout the medullary reticular formation at these levels of the medulla.

\section{Expression of TRPM4 and TRPC3 channels in glutamatergic and glycinergic neurons}

In the transgenic mouse strains with Cre-dependent, cell type-specific expression of tdTomato fluorescent protein, we established by immunolabeling that TRPM4 and TRPC3 channels are present in both glutamatergic and glycinergic neurons within the pre-BötC, as well as other ventral medullary respiratory-related regions examined (BötC, rVRG). The majority of tdTomato-labeled preBötC glutamatergic neurons ( $73 \%, n=1487 / 2049$ cells) examined were colabeled by TRPM4 channel antibody in medullary sections from VgluT2-tdTomato mice (Fig. 2A). In sections from other VgluT2-tdTomato mice $(n=2)$, $71 \%$ ( $n=589 / 826)$ of tdTomato-labeled pre-BötC glutamatergic neurons were labeled by TRPC 3 channel antibody (not shown). We also examined immunolabeling of TRPM4 or TRPC3 channels in sets of glutamatergic neurons within the BötC and rVRG regions. The majority of tdTomato-labeled glutamatergic neurons expressed TRPM4 and TRPC 3 channels in the BötC [63\% $(n=750 / 1185)$ and $70 \%$ ( $n=372 / 527)$ of neurons examined, respectively] and rVRG [74\% $(n=830 / 1120)$ and 62\% $(n=165 / 265)]$.

Because glycinergic neurons are also functional components of respiratory pattern generation circuits within the pre-BötC, BötC, and rVRG regions (Morgado-Valle et al., 2010; Richter and Smith, 2014; Shevtsova et al., 2014), we also examined immunolabeling of TRPM4 and TRPC3 channels in glycinergic neurons in these regions in GlyT2-tdTomato mice ( $n=2$ each). In the pre-BötC, $50 \%$ $(n=340 / 674)$ of the GlyT2-positive neurons examined were immunolabeled with TRPM4 channel antibodies (Fig. $2 B)$, whereas $35 \%(n=171 / 486)$ of GlyT2-positive neurons were labeled by TRPC 3 channel antibodies. In the BötC, $67 \%(n=633 / 942)$ and $65 \%(n=387 / 587)$ of GlyT2-tdTomato-labeled neurons were, respectively, colabeled by TRPM4 and TRPC3 channel antibodies, whereas in the rVRG region only $27.4 \%(79 / 288)$ and 


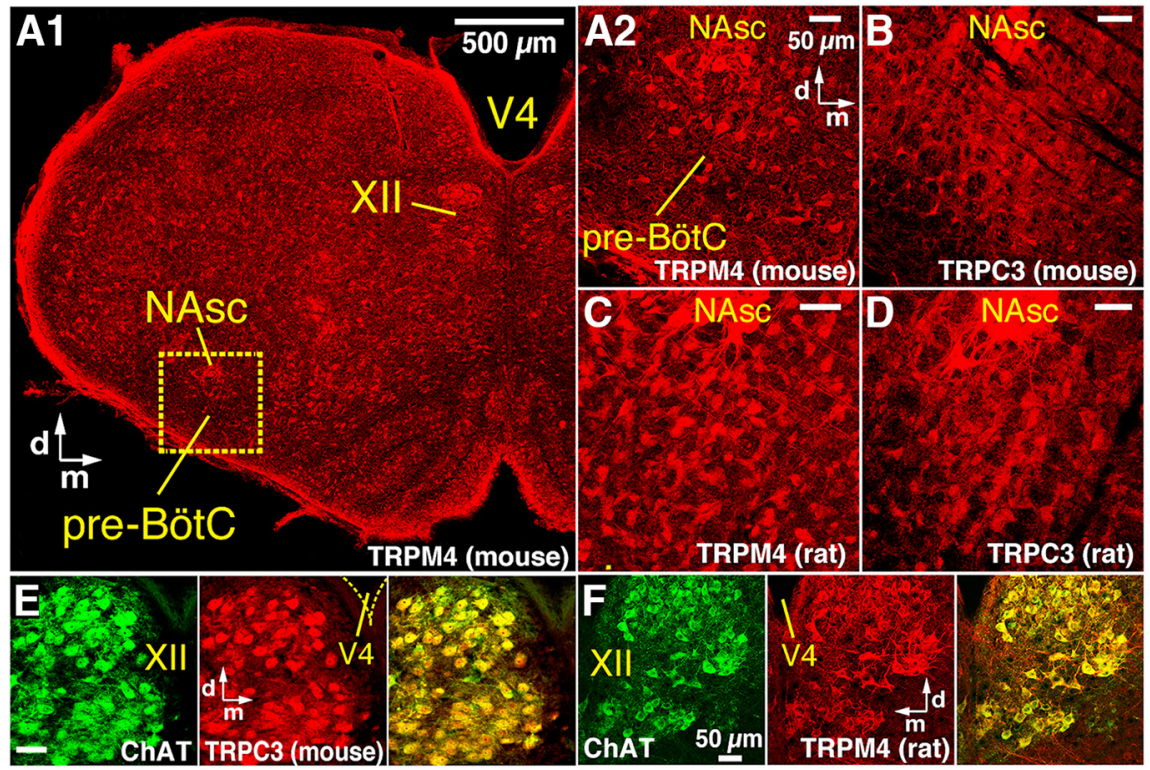

Figure 1. Immunolabeling of TRPM4 and TRPC3 channels in pre-BötC neurons and motoneurons. $\boldsymbol{A}, \boldsymbol{B}$, Confocal fluorescence microscopy images of coronal sections from neonatal mouse medulla at the level of the pre-BötC, showing widely distributed neuronal labeling by TRPM4 (A1, low magnification; $\boldsymbol{A 2}$, higher magnification of dashed box in $\boldsymbol{A 1})$ and TRPC3 (B) channel antibodies (red) within the pre-BötC region and motoneurons within the semicompact division of nucleus ambiguus (NAsc). Note extensive antibody labeling also outside of these regions $(\boldsymbol{A} 1)$. $\boldsymbol{C}, \boldsymbol{D}$, Confocal images of the pre-BötC region from adult rats, showing neuronal labeling by TRPM4 $(\boldsymbol{C})$ and TRPC3 $(\boldsymbol{D})$ channel antibodies within the pre-BötC and labeling of NAsc motoneurons. $\boldsymbol{E}, \boldsymbol{F}$, Confocal images of the hypoglossal (XII) motor nucleus on one side of the medulla at the level containing the pre-BötC from neonatal rat (E) and mouse $(\boldsymbol{F})$, showing TRPM4 $(\boldsymbol{E})$ and TRPC3 $(\boldsymbol{F})$ channel antibody labeling (red) of XII motoneurons identified by ChAT immunolabeling (green). Merged images (right in $\boldsymbol{E}$ and $\boldsymbol{F}$ ) show antibody colabeling of XII motoneurons. $\boldsymbol{A}-\boldsymbol{D}$ have the same dorso-medial anatomic orientation. d, dorsal; $\mathrm{m}$, medial; V4, 4th ventricle.

$15.5 \%(31 / 200)$, respectively, of these neurons were colabeled.

\section{TRPM4 and TRPC3 channel mRNA in glutamatergic, glycinergic/GABAergic pre-BötC inspiratory neurons and cranial motoneurons detected by single-cell multiplex RT-PCR}

To confirm that respiratory neurons express TRP channels, we probed for TRPM4 and TRPC 3 channel mRNA in single functionally identified pre-BötC inspiratory neurons in rhythmically active in vitro medullary slice preparations from WT neonatal rats and mice. Pre-BötC inspiratory neurons were identified by imaging neuronal $\mathrm{Ca}^{2+} \mathrm{dy-}$ namics with OGB at depths up to $150 \mu \mathrm{m}$ in these slices, in which inspiratory neurons exhibit rhythmic $\mathrm{Ca}^{2+}$ fluorescence transients in phase with the inspiratory XII nerve activity (Koizumi et al., 2013). Under current-clamp recording, all optically identified pre-BötC inspiratory neurons exhibited spike discharge synchronized with rhythmic XII activity. In the cytoplasm harvested from these neurons $(n=41$ neurons in total; $n=33$ from 8 rat slices and $n=8$ from 3 mouse slices) during whole-cell recording, we probed for TRPM4 and TRPC 3 channel mRNA as well as VgluT2, GlyT2, and/or GAD67 mRNA to identify neuronal transmitter phenotype (Fig. 3). Only neurons with clean negative controls from "mock harvests" in the slice and appropriate positive controls (see Methods) were used for the analysis. In this sample, we identified 32 excitatory pre-BötC inspiratory neurons expressing only VgluT2 mRNA (28 neurons from rat slices; 4 neurons from mouse slices) and 9 inhibitory neurons expressing either GlyT2 mRNA only ( $n=1$ each from rat and mouse slices), GAD67 mRNA only ( $n=1$ each from rat and mouse), or coexpression of GlyT2 and GAD67 mRNA ( $n=3$ from rat and $n=2$ from mouse slices), a phenotype previously documented for pre-BötC inhibitory interneurons (Koizumi et al., 2013). No VgluT2 mRNA was detected in these inhibitory neurons. Most of the TRP channel mRNApositive pre-BötC inspiratory neurons in this sample were glutamatergic $(n=32 / 41,78 \%)$, and almost half of these excitatory neurons $(n=15 / 32,47 \%)$ coexpressed both TRPM4 and TRPC 3 mRNA, while other excitatory neurons expressed either TRPM4 mRNA only $(n=5)$ or TRPC3 mRNA only $(n=12)$. Inhibitory pre-BötC inspiratory neurons also expressed TRPM4 mRNA only $(n=2)$, TRPC3 mRNA only $(n=5)$, or both TRPM4 and TRPC3 mRNA $(n=2)$.

We also analyzed expression of TRPM4 and TRPC3 channel mRNA in NA and XII motoneurons identified electrophysiologically as inspiratory motoneurons from whole-cell recording in rhythmically active slice preparations from both neonatal rats $(n=4)$ and mice $(n=2)$. We identified coexpression of TRPM4 and TRPC3 channel mRNA in all NA ( $n=5$ motoneurons in total; $n=2$ from rats and $n=3$ from mice) and XII ( $n=10$ total; $n=7$ from rats and $n=3$ from mice) inspiratory motoneurons sampled. Thus, these mRNA expression patterns are consistent with our results from immunolabeling demonstrating prominent TRPM4 and TRPC3 channel antibody labeling in all NA and XII motoneurons, and are consistent with 

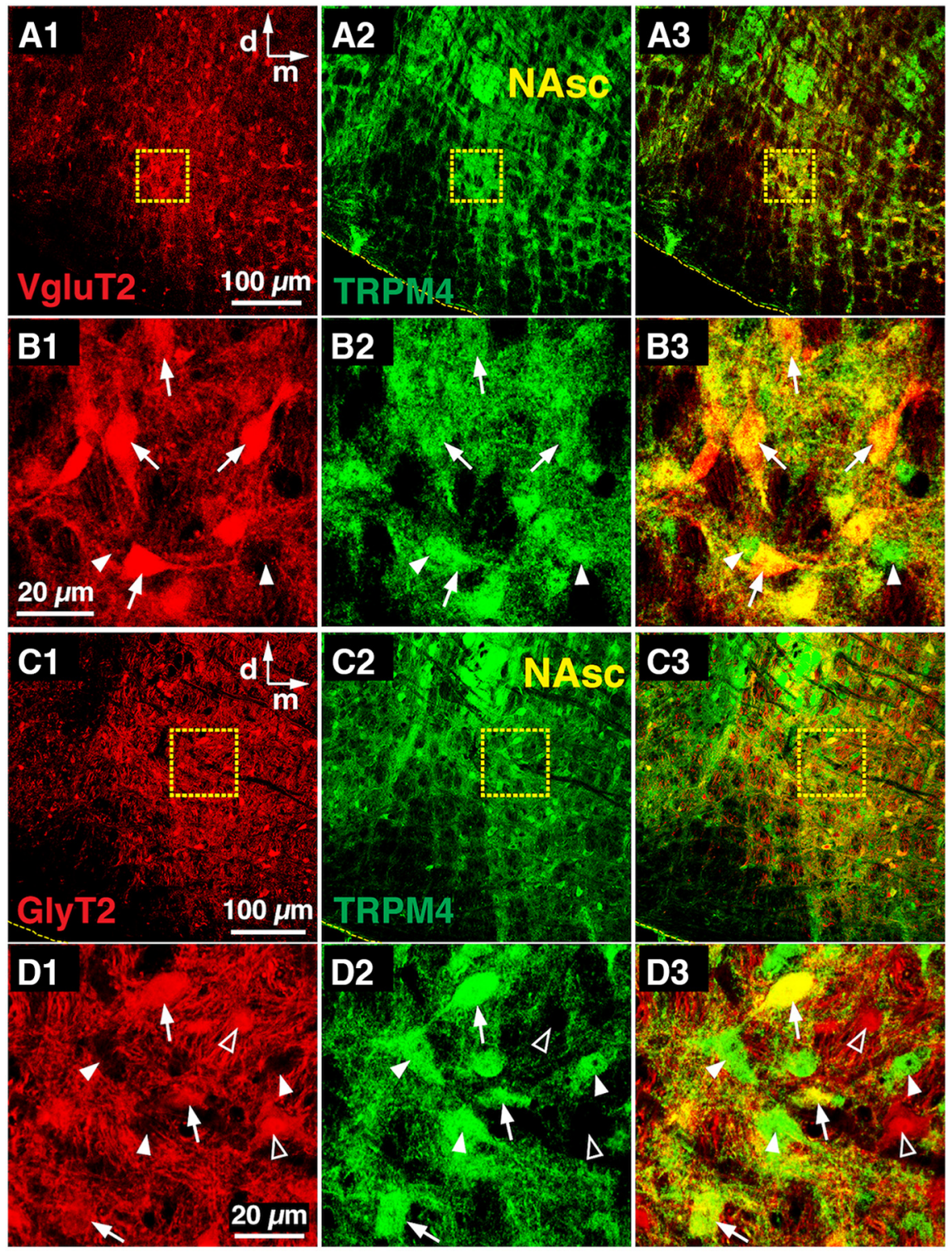

Figure 2. Glutamatergic and glycinergic pre-BötC neurons express TRPM4 channels. $\boldsymbol{A}$, Confocal fluorescence microscopy single plane images of a coronal section of the medulla at the level of the pre-BötC ventral to NAsc from adult VgluT2-tdTomato transgenic mouse, showing Cre-dependent tdTomato-labeled glutamatergic neurons (red, A1), TRPM4 antibody-labeled neurons (green, A2) throughout the pre-BötC, and their merged image (A3). B. Single optical plane images of VgluT2 positive (B1) and TRPM4 (B2) immunolabeled neurons in the pre-BötC subregion marked by dashed box in $\boldsymbol{A}$. Merged image (B3) shows that a majority of VgluT2-positive pre-BötC neurons were colabeled with TRPM4 antibody (white arrows) along with TRPM4 antibody-positive, but VgluT2-negative, neurons (arrowheads). C, Confocal images of the pre-BötC region from adult GlyT2-tdTomato transgenic mouse showing Cre-conditional tdTomato-labeled glycinergic neurons (red, C1), TRPM4 antibody-labeled neurons (green, C2), and the merged image (C3). D. Single optical plane images of GlyT2-positive (D1) and TRPM4 immunolabeled neurons (D2) in the pre-BötC area marked by dashed box in $\boldsymbol{C}$. Merged image (D3) shows GlyT2-positive pre-BötC neurons colabeled with TRPM4 antibody (white arrows) along with TRPM4 antibody-positive, but GlyT2-negative, neurons (arrowheads) as well as TRPM4 antibody-negative, GlyT2-positive neurons (open arrowheads). All images have the same dorso-medial anatomic orientation. NAsc, semi-compact division of nucleus ambiguus; $d$, dorsal; $m$, medial.

previous results showing TRPM4 channel mRNA in lasercaptured XII motoneurons (Alvares et al., 2014).

\section{Perturbations of inspiratory motor output in vitro by pharmacological inhibitors of TRPM4 and TRPC3 channels}

The expression of TRPM4 and TRPC 3 channel mRNA in identified pre-BötC inspiratory neurons and inspiratory cranial motoneurons, and the extensive antibody labeling of these channels in the pre-BötC region and adjacent respiratory-related regions as well as motor nuclei, suggest possible functional roles of these channels in rhythm and motor pattern generation. To test for functional endogenous activity of these channels in the rhythmically active neonatal rat and mouse slice preparations in vitro, 


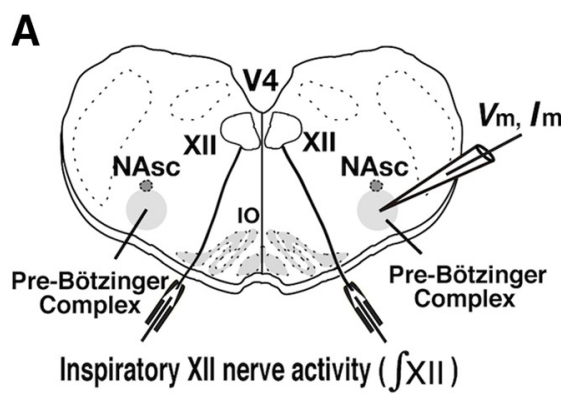

C1 Excitatory neuron
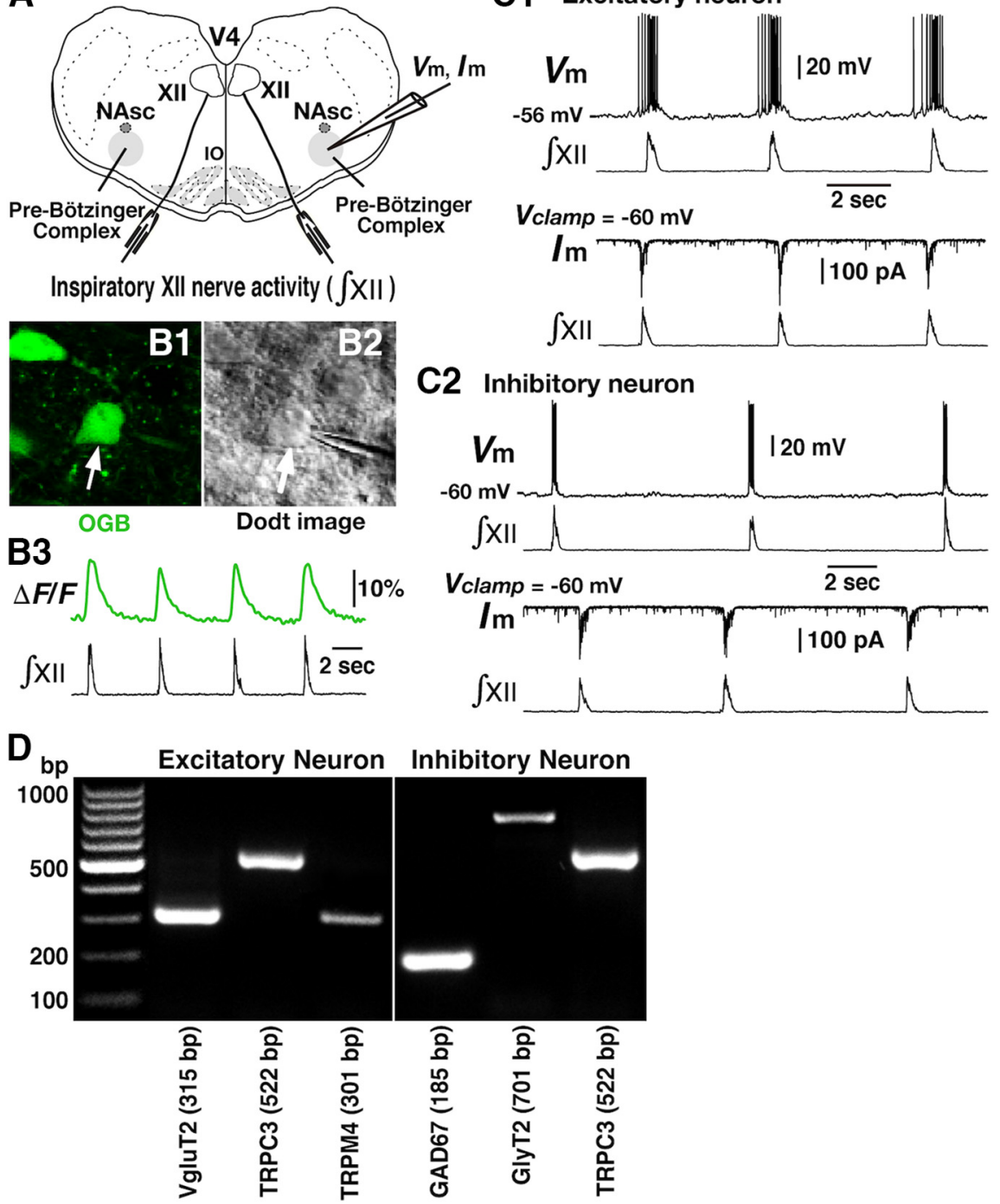

Figure 3. Expression of TRPM4 and TRPC3 channel mRNA in glutamatergic and glycinergic/GABAergic pre-BötC inspiratory neurons. $\boldsymbol{A}$, Overview of experimental in vitro neonatal rat rhythmic slice preparation showing whole-cell patch-clamp recording from the pre-BötC inspiratory neurons and suction electrode recordings from hypoglossal (XII) nerves to monitor inspiratory activity. NAsc, semicompact division of nucleus ambiguus; V4, fourth ventricle. $\boldsymbol{B}$, Two-photon single optical plane images of pre-BötC inspiratory neuron (arrow) targeted for whole-cell recording and subsequent harvesting of cytoplasm, showing $\mathrm{Ca}^{2+}$-sensitive dye (OGB) labeling (B1) and Dodt structural image (B2). B3, Identification of inspiratory neuron by verifying that the $\mathrm{Ca}^{2+}$ fluorescence signals in real time are synchronized with integrated inspiratory XII nerve activity $\left(\int \mathrm{XII}\right)$. $\mathbf{C 1}$, Current-clamp recording (upper traces) from excitatory pre-BötC inspiratory neuron in B illustrates inspiratory bursts synchronized with $\int$ XII. Under voltage-clamp (lower traces), the same neuron exhibited rhythmic inward synaptic currents synchronized with $\int$ XII. This neuron was shown to be excitatory (VgluT2expressing) by post hoc single-cell RT-PCR (below). C2, Current-clamp recording (upper traces) and voltage-clamp recording (lower traces) from inhibitory pre-BötC inspiratory neuron illustrating inspiratory bursts and rhythmic inward synaptic currents synchronized with $\int$ XII. This neuron was shown to be inhibitory (co-expression of GlyT2 and GAD67 mRNA) by post hoc single-cell RT-PCR (see below). $\boldsymbol{D}$, Representative electrophoresis gel generated by single-cell multiplex RT-PCR from mRNA in cytoplasm harvested during whole-cell recording from two electrophysiologically identified pre-BötC inspiratory neurons (C1 and C2) in neonatal rat slices. In addition to cDNA probes for TRPM4 and TRPC3 channel mRNA, probes for vesicular glutamate transporter type 2 (VgluT2), glycine transporter type 2 (GlyT2), and glutamatic acid decarboxylase 67 (GAD67) mRNA were used to identify excitatory or inhibitory neuronal phenotypes, examples of which are shown. Expected numbers of base pairs (bp) for reaction products are indicated. Assays for both of these neurons had clean negative controls from "mock harvests" in the slice and appropriate positive controls from 100 pg total rat brain RNA run as RT template (not shown, see Materials and Methods).

we initially analyzed perturbations of the inspiratory rhythm and burst amplitude/duration of integrated XII inspiratory motor output after bath-application of the TRPM4 channel inhibitor 9-phenanthrol, the TRPC3 chan- nel inhibitor Pyr3, and the $I_{\text {CAN }}$ blocker FFA. In preliminary experiments, we determined that 9-phenanthrol (10-50 $\mu \mathrm{M})$, Pyr3 $(10-50 \mu \mathrm{M})$, and FFA $(20-75 \mu \mathrm{M})$ progressively reduced the amplitude of XII inspiratory activity and, in 
some cases, could completely eliminate XII inspiratory motor output at $50 \mu \mathrm{M}$ in our rat and mouse slice preparations. We therefore routinely used a single application of $50 \mu \mathrm{M}$ for these drugs as a near upper bound for circuit activity perturbations.

With $50 \mu \mathrm{M}$ 9-phenanthrol, for both rat and mouse slices, we consistently found large perturbations of integrated XII burst amplitude without significant perturbations of inspiratory burst frequency $\left(f_{R}\right)$ relative to control values. Fig. $4 A$ shows an example from an individual experiment, as well as the averaged, normalized time course of the reduction in XII amplitude and nonsignificant perturbation of normalized $f_{\mathrm{R}}$ as burst amplitude reached a quasi-steady state value from a set of rat slices $(n=6)$. The reduction of peak inspiratory burst amplitude for the group of experiments $(57 \pm 7 \%$ reduction in mean amplitude; $p=0.03$ ) was accompanied by a significant reduction of inspiratory burst duration $\left(T_{1}, 29 \pm 8 \%\right.$ reduction; $p=0.03$ ) at the defined endpoint of the time series at 32.6 $\pm 8.1 \mathrm{~min}$. This change in amplitude and $T_{1}$ was accompanied by a nonsignificant change of $f_{\mathrm{R}}$ (reduction by $6 \pm$ $9 \%, p=0.31$ ) due to a small, insignificant increase in $T_{\mathrm{E}}$ (15 $\pm 12 \%$ increase; $p=0.44)$. Similarly, in mouse slices $(n=6$, Fig. $5 A)$ the significant reduction in peak integrated XII amplitude and $T_{1}$ from control values was $60 \pm 8 \%(p$ $=0.03)$ and $28 \pm 3 \%(p=0.03)$, respectively, without a significant change in $f_{\mathrm{R}}(8 \pm 9 \%$ increase, $p=0.44)$ or $T_{\mathrm{E}}$ $(2 \pm 10 \%$ decrease, $p=0.69)$ at $20.28 \pm 3.4 \mathrm{~min}$ after bath-application of $50 \mu \mathrm{M} 9$-phenanthrol. In these pharmacological experiments, as well as those described below, we obtained only partial recovery of XII burst amplitude after up to $1 \mathrm{~h}$ of continuous drug washout.

In both rat and mouse slices (Figs. $4 B$ and $5 B$ ), bathapplication of the TRPC3 channel inhibitor Pyr3 $(50 \mu \mathrm{M})$ also reduced the integrated XII amplitude and $T_{1}$ without significant perturbations of $f_{\mathrm{R}}$. The Pyr3-induced reduction in group $(n=6)$ mean XII amplitude and $T_{1}$ in rat slices (Fig. $4 B$ ) was respectively $73 \pm 6 \%(p=0.03)$ and $27 \pm$ $1 \%(p=0.03)$, while the group mean $f_{\mathrm{R}}$ was unchanged $(0$ $\pm 7 \%$ change from control, $p=0.85$ ) at the defined endpoint values at $38.8 \pm 6.6 \mathrm{~min}$. In mouse slices (Fig. $5 B)$, the reduction in group $(n=6)$ XII mean amplitude and $T_{1}$ was $47 \pm 6 \%(p=0.03)$ and $27 \pm 6 \%(p=0.03)$, while the mean $f_{\mathrm{R}}$ increased nonsignificantly above control values $(20 \pm 9 \%$ increase, $p=0.16)$ at the endpoint $28.2 \pm$ 3.9 min after bath-applied $50 \mu \mathrm{M}$ Pyr3.

Comparable data sets for perturbations of XII amplitude and $T_{1}$ after bath application of the $I_{\text {CAN }}$ blocker FFA (50 $\mu \mathrm{M})$ are shown, respectively, for rat and mouse slices in Figs. $4 C$ and $5 C$. FFA reduced the group $(n=6)$ mean XII amplitude and $T_{1}$, respectively, in rat slices (Fig. $4 C$ ) by 57 $\pm 5 \%(p=0.03)$ and $25 \pm 6 \%(p=0.03)$, while the mean $f_{\mathrm{R}}$ was unchanged $(0 \pm 10 \%$ change from control, $p=$ $1.00)$ at the $28.3 \pm 4.1-$ min endpoint. FFA significantly reduced the group mean $(n=6)$ XII amplitude in mouse slices (Fig. $5 C$ ) by $45 \pm 12 \%$ ( $p=0.03$ ), although $T_{1}$ was not significantly reduced $(5 \pm 1 \%$ reduction, $p=0.06)$ and $f_{\mathrm{R}}$ was nonsignificantly increased $(16 \pm 12 \%, p=$ 0.31) from control values at the quasi-steady state
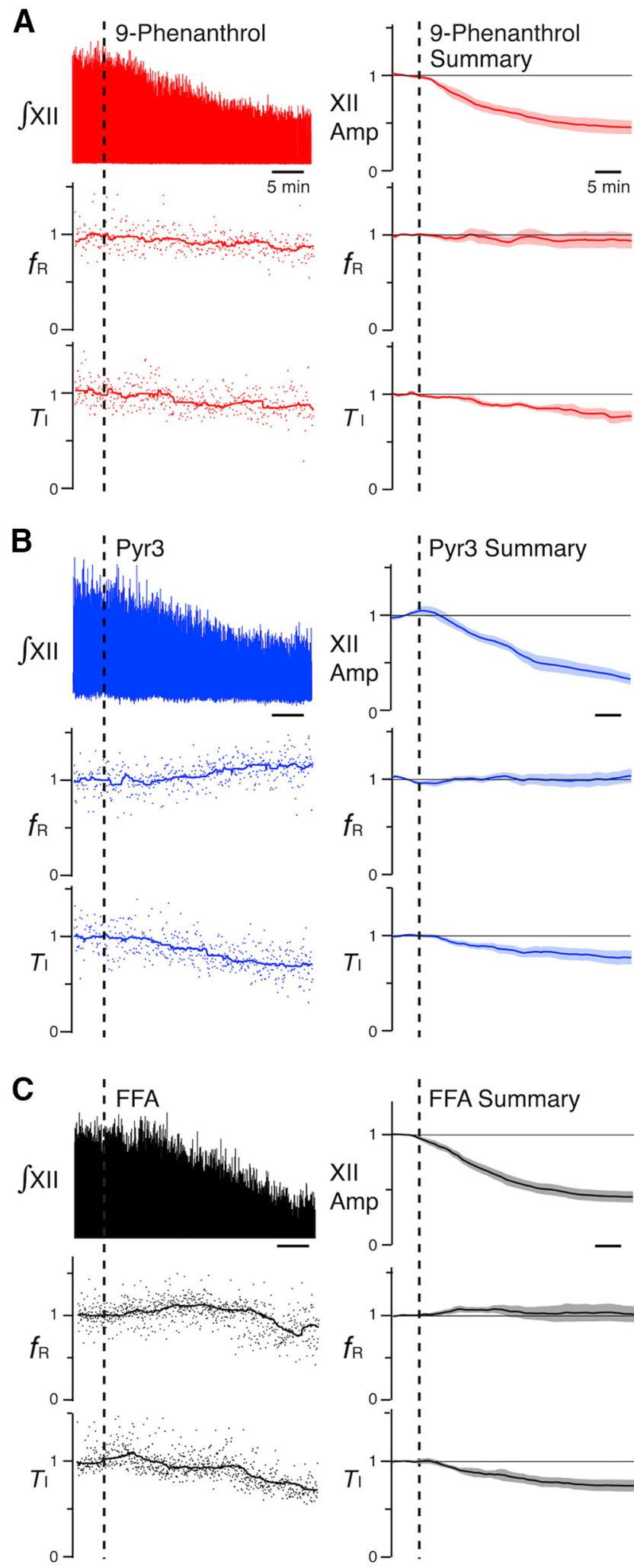

Figure 4. Effects of TRPM4, TRPC3, and $I_{\text {CAN }}$ channel inhibitors on rhythmic hypoglossal (XII) inspiratory activity in neonatal rat medullary slice preparations in vitro. $\boldsymbol{A}-\boldsymbol{C}$, Left panels illustrate time courses of integrated XII burst activities ( $\int$ XII), inspiratory frequency $\left(f_{R}\right)$, and inspiratory activity time $\left(T_{1}\right)$ during application of each inhibitor $(\boldsymbol{A}, 50 \mu \mathrm{m}$ 9-phenanthrol; $\boldsymbol{B}, 50 \mu \mathrm{M}$ Pyr3; $\boldsymbol{C}, 50$ $\mu \mathrm{M}$ FFA) from representative individual experiments (dots: in- 
continued

stantaneous $f_{\mathrm{R}}$ and $T_{1}$; solid lines: running median). Right panels show group summary of mean time course (solid lines) and SEM (light-color bands) of normalized integrated XII amplitude (XII Amp), $f_{\mathrm{R}}$, and $T_{1}$ after drug administration ( $n=6$ slice preparations each). Time of drug administration is indicated by vertical dashed lines.

reached $17.1 \pm 2.3 \mathrm{~min}$ after bath application of $50 \mu \mathrm{M}$ FFA.

We also performed control experiments $(n=6$ each in rats and mice), in which the XII nerve activity was recorded for 60 min without application of any pharmacological agents, and found no significant changes in inspiratory burst amplitude of XII nerve activity $(100 \pm 3 \%$ in rats and $99 \pm 4 \%$ in mice compared with the control value at $60 \mathrm{~min} ; p=0.36$ in rats and $p=0.36$ in mice).

In addition to establishing that 9-phenanthrol, Pyr3, and FFA did not significantly change $f_{\mathrm{R}}$ while causing large reductions in XII discharge amplitude, we also determined that these amplitude perturbations were not accompanied by significant changes in variability of the inspiratory rhythm. Short-term (burst-to-burst, SD1, Fig. 6) and longerterm (SD2) period variability, quantified for $T_{\text {TOT }}$ from Poincaré maps (see Methods) for the time series analyzed as the amplitude perturbations approached quasi-steady state values for rat or mouse slices, were not significantly different from control values for each channel inhibitor (Fig. 6). For rat slices $(n=6)$, SD1 $=123 \pm 12 \%(p=$ $0.31), 104 \pm 10 \%(p=1.00)$, and $116 \pm 19 \%(p=0.31)$ of control values for 9-phenanthrol, Pyr3, and FFA, respectively; SD2 $=138 \pm 17 \%(p=0.69), 98 \pm 13 \%(p=$ $0.44)$, and $124 \pm 29 \%(p=0.56)$, respectively, for these inhibitors. For mouse slices $(n=6)$, SD1 $=125 \pm 02 \%$ $(p=0.09), 97 \pm 12 \%(p=0.84)$, and $89 \pm 7 \%(p=0.56)$ of control values for 9-phenanthrol, Pyr3, and FFA, respectively; SD2 $=107 \pm 11 \%(p=0.09), 110 \pm 12 \%(p$ $=0.84)$, and $93 \pm 7 \%(p=0.56)$ of control values for these inhibitors. Similarly, the mean coefficient of variation
(CV) for the time series for each inhibitor was not significantly different from control values (Fig. 6). CV $=120 \pm 12 \%$ ( $p=$ $0.22), 99 \pm 8 \%(p=1.00)$, and $120 \pm 21 \%(p=0.56)$ of control values for 9-phenanthrol, Pyr3, and FFA, respectively, for rat slices, and CV $=113 \pm 7 \%(p=0.16), 107 \pm$ $10 \%(p=1.00)$, and $98.0 \pm 5 \%(p=0.69)$ of control for 9-phenanthrol, Pyr3, and FFA, for mouse slices.

\section{Simultaneous perturbations within pre-BötC excitatory circuits and hypoglossal motor output in vitro with bath-applied channel inhibitors}

Because TRPM4 and TRPC3 channels were expressed in pre-BötC and XII inspiratory neurons, reductions in XII activity amplitude could potentially reflect reduced excitability of XII motoneurons. To establish contributions of pre-BötC neurons, we analyzed correlations between the observed perturbations in motor output amplitude and perturbations of pre-BötC excitatory neuron population activity. For these experiments, we used in vitro rhythmic slices from VgluT2-GCaMP6 transgenic mice expressing the fluorescent $\mathrm{Ca}^{2+}$-sensor GCaMP6f in glutamatergic neurons to image neuronal and population activity within the pre-BötC (see Methods) during simultaneous recording of XII motor output (Fig. 7). Application of $50 \mu \mathrm{M}$ 9-phenanthrol, Pyr3, or FFA to the transgenic mouse slice preparations significantly decreased the amplitude of the field $\Delta F$ (i.e., $F-F_{0}$ ), indicating reduced excitatory neuron population activity, which typically reached quasi-steady state by 20 min after drug application (e.g., see Fig. 7D) and was accompanied by a significant reduction in amplitude of integrated XII activity. The time-dependent reductions of $\Delta \mathrm{F}$ and $\int \mathrm{XII}$ amplitudes (XII Amp) were linearly correlated (see regression lines and Pearson correlation coefficients for data in Figs. $7 D$ and $8 C$ ). The reductions in amplitude of field $\Delta F$ normalized to control values with 9-phenanthrol $(n=5)$, Pyr3 $(n=5)$, and FFA $(n=4)$ were $49 \pm 7 \%, 52 \pm 6 \%$, and $39 \pm 8 \%$, respectively, at $20 \mathrm{~min}$ after drug administration (Fig. 8B). These amplitude re-
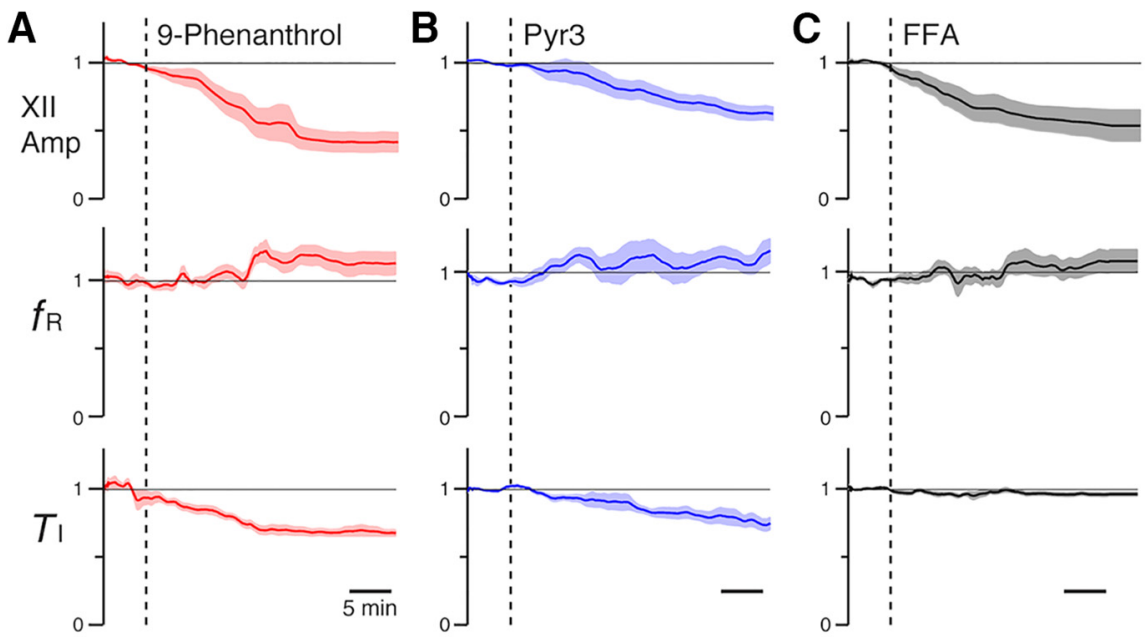

Figure 5. Effects of TRPM4 $(\boldsymbol{A})$, TRPC3 $(\boldsymbol{B})$, and $I_{\text {CAN }}(\boldsymbol{C})$ channel inhibitors on rhythmic hypoglossal (XII) activities in neonatal mouse medullary slice preparations in vitro. $\boldsymbol{A}-\boldsymbol{C}$, Mean time course (solid line) and SEM (light-color band) of the amplitude of normalized integrated XII burst activities (XII Amp), inspiratory frequency $\left(f_{\mathrm{R}}\right)$, and inspiratory activity time $\left(T_{1}\right)$ during drug application $(n=6$ slices each). Time of drug administration ( $\boldsymbol{A}, 50 \mu \mathrm{M}$ 9-phenanthrol; $\boldsymbol{B}, 50 \mu \mathrm{M}$ Pyr3; $\boldsymbol{C}, 50 \mu \mathrm{M}$ FFA) is indicated by vertical broken lines. 
A
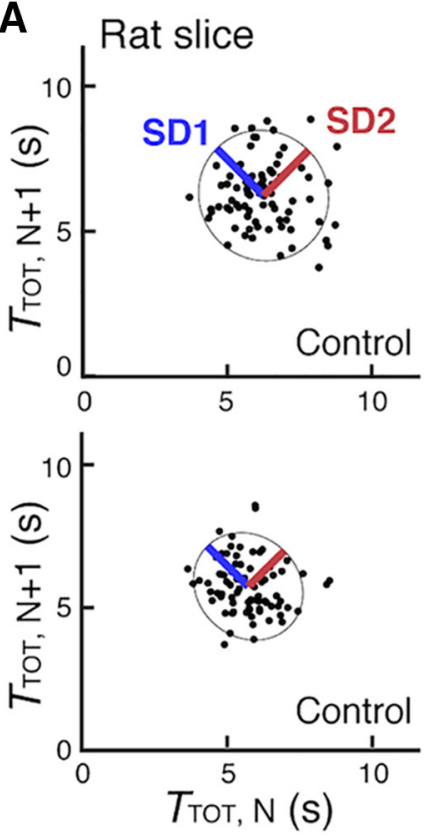
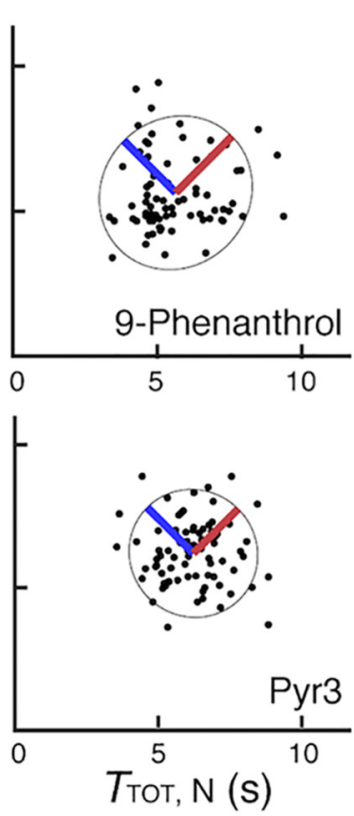

B
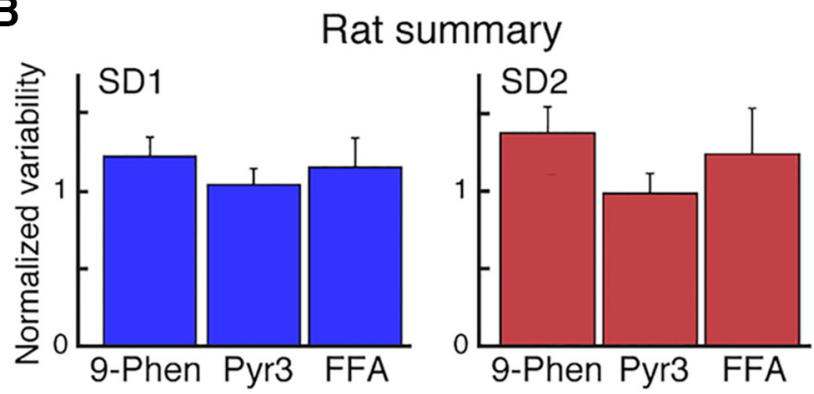

C
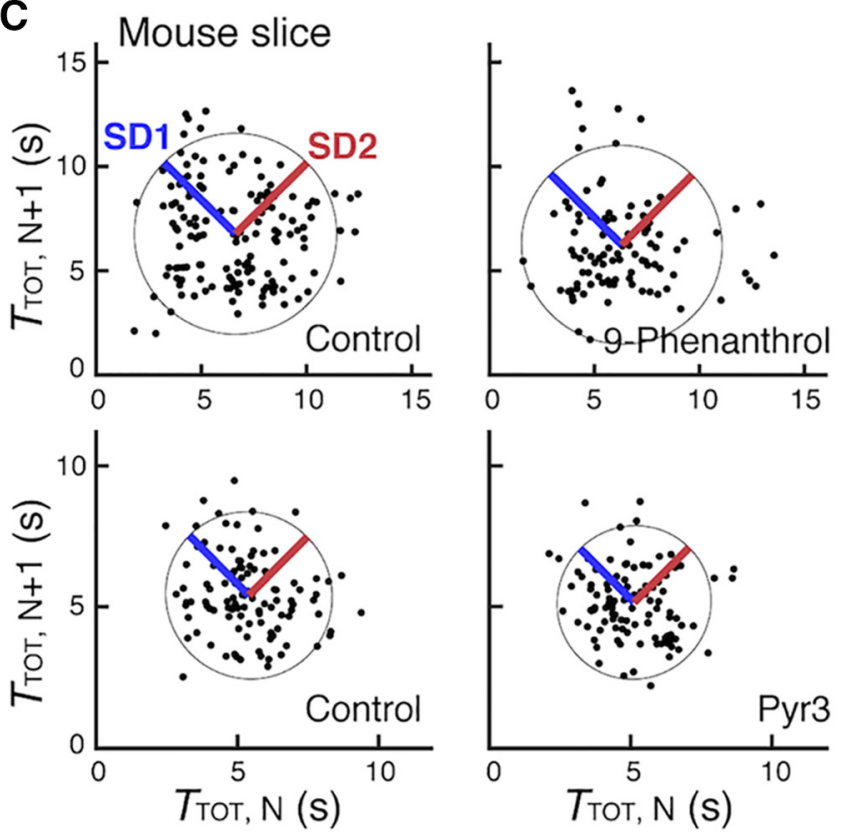

D
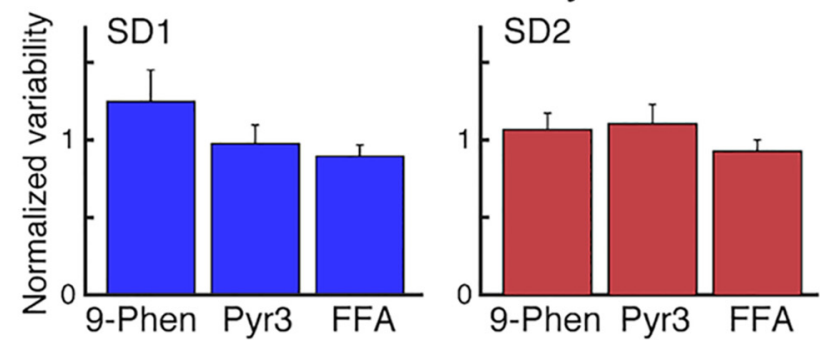

Figure 6. Pharmacological inhibition of TRPM4, TRPC3, and $I_{\text {CAN }}$ does not affect variability of inspiratory rhythm in vitro. $\boldsymbol{A}$, $\boldsymbol{B}$, Poincaré maps $(\boldsymbol{A})$ from a representative in vitro rat slice experiment with inhibition of TRPM4 and TRPC3 channels, respectively, by 9-phenanthrol (9-Phen in $\boldsymbol{B}$ ) and Pyr3, illustrating geometric fits of XII inspiratory period data and representations of short-term (SD1) and long-term (SD2) variability measures (see Methods for definitions), and summary data (B) for the analyzed group ( $n=6$ each). $\boldsymbol{C}$, $\boldsymbol{D}$, Equivalent sets of data from in vitro mouse slice experiments. Variability measures normalized to control values were not statistically significant in all cases in $\boldsymbol{B}$ and $\boldsymbol{D}$. $T_{\text {ТОТ }}$, respiratory period.

ductions were significant over time in all cases: for 9-phenanthrol, the Spearman correlation coefficient $r_{s}=$ $-0.88(p=0.017)$, for Pyr3 $r_{s}=-0.63(p=0.0028)$, and for FFA $r_{s}=-0.72(p=0.031)$. The $\Delta F$ amplitude perturbations were accompanied by nonsignificant changes in normalized $f_{\mathrm{R}}$ for the imaged population (Fig. 8B) of $14 \pm 6 \%,-17 \pm 18 \%$, and $5 \pm 6 \%$ at $20 \mathrm{~min}$ with 9-phenanthrol $\left(r_{s}=0.14, p=0.52\right)$, Pyr3 $\left(r_{s}=-0.11, p=\right.$ $0.60)$, and FFA $\left(r_{s}=-0.30, p=0.22\right)$, respectively. We performed control experiments (Fig. $8 A, n=5$ mice) to test for possible photobleaching or time-dependent changes in population activity, in which calcium imaging was performed without any drug application with exactly the same protocol of image acquisition as the pharmacological experiments. The results showed that there were no significant changes in the pre-BötC field $\Delta F(102 \pm 3 \%$ of control at $20 \mathrm{~min}$, Spearman correlation coefficient $r_{s}=$ $0.11 ; p=0.62)$, integrated XII amplitude (99 $\pm 3 \%, r_{s}=$ $-0.078 ; p=0.74)$, and respiratory frequency $(100 \pm 2 \%$, $r_{s}=0.092 ; p=0.70$ ).
We also tracked perturbations of $\mathrm{Ca}^{2+}$ transients of individual pre-BötC glutamatergic neurons (cell $\Delta F$ ) in relation to population-level (field $\Delta F$ ) perturbations (Figs. 9 and 10). The reduction in mean amplitude of cell $\Delta F$ (normalized to control amplitudes) for sets of imaged neurons with inspiratory $\mathrm{Ca}^{2+}$ transients was correlated with the reduction in normalized field $\Delta F$ over time after drug application. We note that some inspiratory neurons in these experiments exhibited normalized $\Delta F$ values that were unaffected or increased during drug application (outlier points in Fig. 10). Regardless, the mean cell $\Delta F$ for the entire group of inspiratory neurons analyzed was strongly correlated (see identity lines in Fig. 10) with the mean field $\Delta F$.

\section{Perturbations of pre-BötC activity and motor output by TRPM4 and TRPC 3 channel inhibitors in perfused brainstem-spinal cord preparations in situ}

We analyzed contributions of endogenously active TRPM4 and TRPC3 channels to rhythm and motor pattern generation in mature rat and mouse arterially perfused 

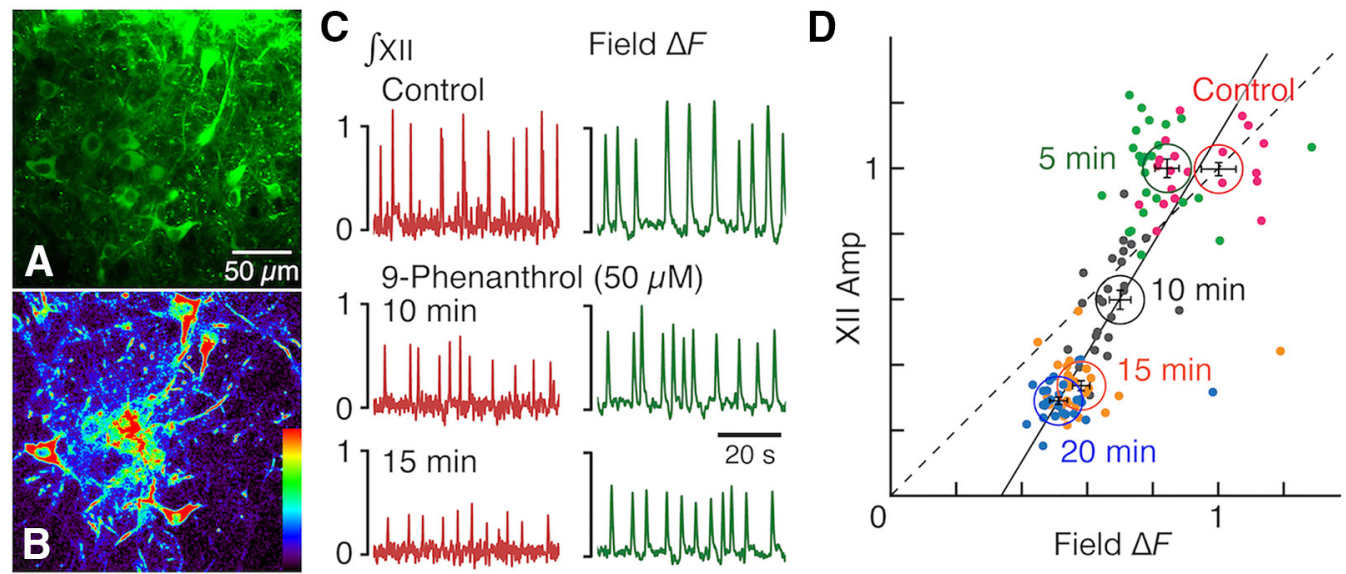

Figure 7. Perturbations of imaged pre-BötC inspiratory $\mathrm{Ca}^{2+}$ activity and electrophysiologically recorded hypoglossal motor output in the VgluT2-GCaMP6f transgenic mouse in vitro slice during application of TRPM4 channel inhibitor. A, Example of two-photon single optical plane image showing Cre-dependent GCaMP6f expression in pre-BötC glutamatergic neurons. $\boldsymbol{B}$, Background subtracted $\left(F-F_{0}\right) \Delta F$ image showing increased GCaMP6f fluorescence of individual neurons for the optical plane shown in $\boldsymbol{A}$. $\boldsymbol{C}$, Integrated inspiratory hypoglossal activity $\left(\int \mathrm{XII}\right)$ and the spatially averaged field GCaMP6f fluorescence transients $(\Delta F)$, quantified as $F-F_{0}$, of the optical plane shown in $\boldsymbol{A}$ during control time, and 10 and 15 min after bath-application of 9-phenanthrol (50 $\left.\mu \mathrm{M}\right)$. $\boldsymbol{D}$, Inspiratory burst-wise correlations of the field fluorescence $\Delta F$ and $\int$ XII amplitudes (XII Amp; colored dots), and their grouped averages (circles with error cross bars: mean values \pm SEM), normalized to their control values, for time windows 5 , 10, 15 , and 20 min after 9-phenanthrol application. Note that the 15- and 20-min point clusters are nearly superimposed, indicating quasi-steady state of the perturbations was achieved by $20 \mathrm{~min}$. The identity line (dashed) and linear regression line (solid; Pearson correlation coefficient $r=0.74$ ) indicate significant correlation between peak field $\Delta F$ and XII Amp.

brainstem-spinal cord preparations in situ to assess functional roles in more intact respiratory circuits generating a three-phase rhythmic activity pattern similar to that in vivo. We analyzed perturbations of extracellularly recorded pre-BötC and PN nerve inspiratory activities as well as VN inspiratory and post-inspiratory (post-I) activities after systemic application of 9-phenanthrol and Pyr3 in the brainstem-spinal cord via perfusion solution.

Perturbations of respiratory pattern and frequency caused by 9-phenanthrol are illustrated by examples in Fig. 11 for rat (Fig. $11 A-C$ ) and mouse (Fig. 11D-F) preparations, which show time courses of the perturbations of normalized amplitudes of integrated PN and VN respiratory motor output and simultaneously recorded pre-BötC inspiratory population activity, and $f_{\mathrm{R}}$ by $\mathrm{PN}$. Mean normalized time courses summarizing the perturbations by 9-phenanthrol and Pyr3 for the groups of rat and mouse preparations analyzed are presented in Fig. 12. In general, these data sets show that systemic administration of 9-phenanthrol (50 $\mu \mathrm{M}$ for $n=6$ rat preparations, and 20-50 $\mu \mathrm{M}$ (pooled) for $n=7$ mouse preparations) reduced the quasi-steady state amplitudes (analyzed at 8 and 14 min, respectively, for rat and mouse preparations) of integrated inspiratory activity of the pre-BötC (rat: $78 \pm$ $16 \%$ decrease, $p=0.03$; mouse: $39 \pm 21 \%$ decrease, $p$ $=0.03$ ), and PN (rat: $77 \pm 16 \%$ decrease, $p=0.03$; mouse: $74 \pm 7 \%$ decrease, $p=0.016$ ). The amplitude of integrated VN inspiratory activity (e.g., Fig. 11) and post-I activity was also strongly reduced (rat post-l: $81 \pm 14 \%$ decrease, $p=0.03$; mouse post-I: $62 \pm 11 \%$ decrease, $p$ $=0.02$ ). Mean normalized inspiratory $f_{\mathrm{R}}$ increased significantly after application of $50 \mu \mathrm{M}$ 9-phenanthrol in both rats and mice (by $49 \pm 15 \%, p=0.03$, and $129 \pm 43 \%$, $p=0.03$, respectively), due to a reduction in expiratory phase duration $\left(T_{\mathrm{E}}\right)$ in the rat in situ preparations by 44.5 $\pm 8.5 \%(p=0.03)$ and mouse preparations by $57 \pm 2.4 \%$ $(p=0.03)$, accompanied by relatively small perturbations of $T_{1}$ (rat: $8 \pm 11 \%$ increase, $p=0.44$; mouse: $24 \pm 3 \%$ decrease, $p=0.03$ ).

Pyr3 (50 $\mu \mathrm{M}, n=8$ rat and 6 mouse preparations) reduced the normalized amplitudes of integrated inspiratory activity of the pre-BötC (rat: $58 \pm 9 \%$ reduction, $p=$ 0.01 ; mouse: $26 \pm 7 \%$ reduction, $p=0.03$ ), and PN (rat: $43 \pm 11 \%$ reduction, $p=0.02$; mouse: $61 \pm 15 \%, p=$ 0.03 ; Fig. 12). The amplitude of integrated VN post-I activity was reduced by $64 \pm 9 \%(p=0.01)$ and $69 \pm$ $10 \%(p=0.03)$ in rat and mouse preparations, respectively. These perturbations were accompanied by a decrease in the group mean $f_{\mathrm{R}}$ by $33 \pm 8 \%(p=0.01)$ from control values in rat preparations, but no significant perturbations of $f_{\mathrm{R}}$ in mouse preparations $(10 \pm 14 \%$ increase, $p=0.44$ ).

\section{Discussion}

Biophysical mechanisms generating and transmitting rhythmic activity within excitatory pre-BötC circuits remain undefined despite extensive experimental and modeling studies investigating possible $\mathrm{Na}^{+}$- and $\mathrm{Ca}^{2+}$-based mechanisms of rhythmic pre-BötC cellular and population-level bursting activity (Rybak et al., 2014). $\mathrm{Ca}^{2+}$ signaling-based theories incorporating $I_{\text {CAN }}$, postulated to be mediated by $\mathrm{Ca}^{2+}$-activated TRPM4 channels (Mironov, 2008; Del Negro et al., 2010), have been proposed. However, TRPM4 and other potentially important TRP channels mediating nonselective cationic currents involved in $\mathrm{Ca}^{2+}$-related signaling such as TRPC3, although proposed, have not been identified in pre-BötC and other 

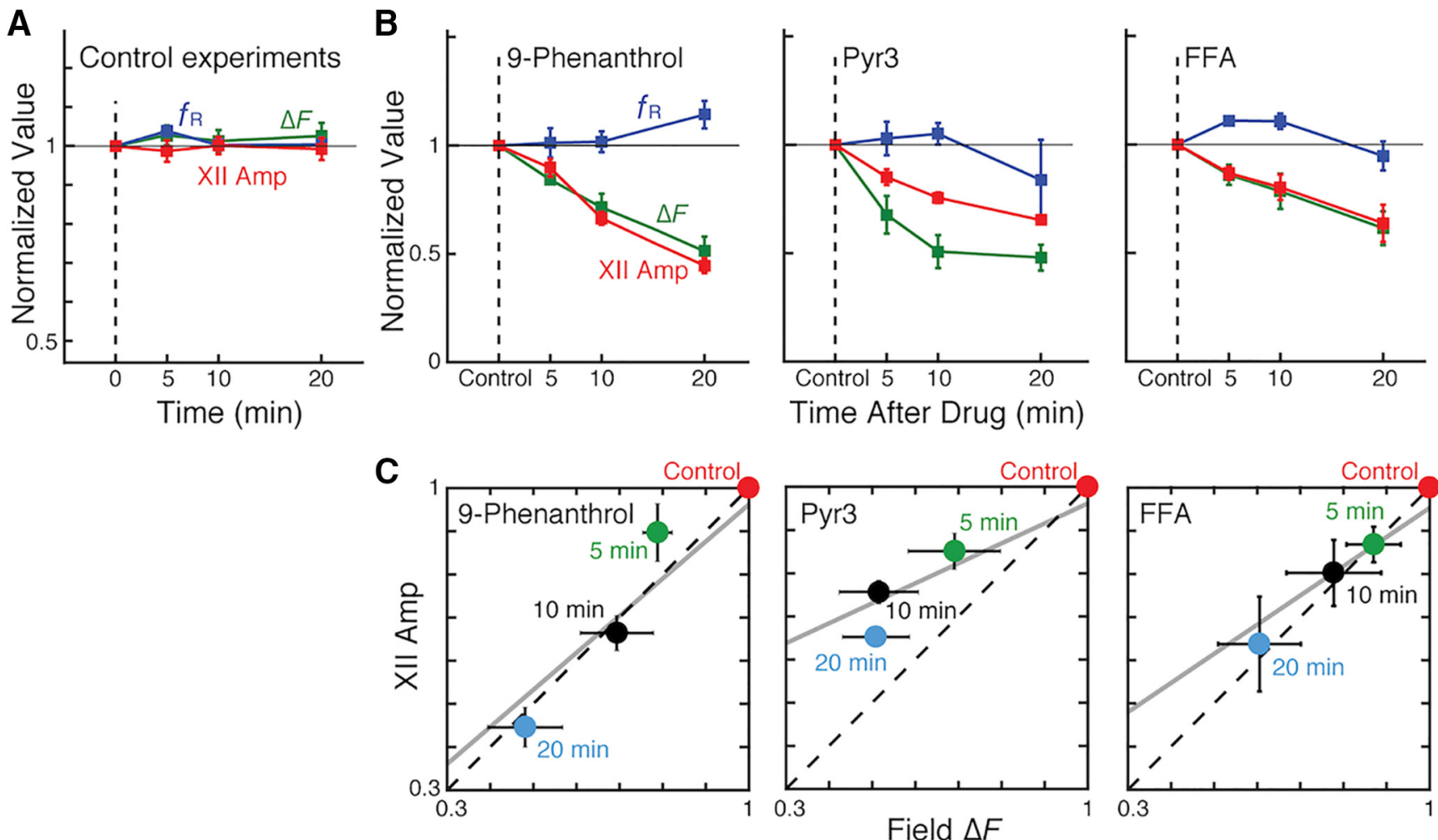

Figure 8. Time-dependent changes in the amplitude of integrated XII inspiratory burst activities (XII Amp) and the field GCaMP6f fluorescence transients $(\Delta F)$ of the pre-BötC glutamatergic population after TRPM4, TRPC3, and $I_{\text {CAN }}$ inhibitors. A, Control experiments ( $n=5$ mice) to test for possible photobleaching and time-dependent changes in population activity, in which calcium imaging was performed without any drug application with exactly the same protocol of image acquisition as the pharmacological experiments. The results (mean normalized values \pm SEM) show that there were no significant changes in the pre-BötC field $\Delta F$ amplitude, XII Amp (normalized to control values), and normalized inspiratory burst frequency $\left(f_{\mathrm{R}}\right)$. $\boldsymbol{B}$, Group summary data (mean normalized values \pm SEM) for 9-phenanthrol, Pyr3, and FFA $(n=5,5$, and 4 , respectively) shows reduction in both XII Amp and the pre-BötC field $\Delta F$ amplitude, while $f_{\mathrm{R}}$ changed nonsignificantly after applying channel inhibitors in all cases. $\boldsymbol{C}$, Time-dependent reductions of XII Amp and field $\Delta F$ amplitudes after drug application were positively correlated (solid lines: linear regression; Pearson linear correlation coefficient for 9-phenanthrol, Pyr3, and FFA: $r=0.864,0.845$, and 0.749, respectively). The linear regression on mean amplitude reduction between XII Amp and field $\Delta F$ for 9-phenanthrol, Pyr3, and FFA yielded corresponding linear models with slopes $m=0.859,0.463$, and 0.676 , and intercepts $b=0.103,0.499$, and 0.277 , respectively. Dashed lines represent the identity line.

respiratory neurons. Furthermore, their functional roles in respiratory circuits have not been clearly defined.

We obtained evidence for TRPM4 and TRPC3 channel mRNA in pre-BötC inspiratory neurons as well as medullary respiratory motoneurons. The pharmacologically induced perturbations of circuit activity by their putative selective channel inhibitors indicate endogenous activity of these channels with major functional roles in formation of inspiratory and post-inspiratory respiratory activity. However, our results indicate that these channels do not contribute to the generation and stability of inspiratory rhythm in pre-BötC circuits. These results therefore do not support previous $\mathrm{Ca}^{2+}$ signaling-based hypotheses incorporating TRPM4// CAN $_{\text {in }}$ pre-BötC neurons as a fundamental mechanism for rhythm generation.

\section{TRPM4 and TRPC3 channels in respiratory neurons}

Our initial survey of neuronal expression of TRPM4 and TRPC3 channels by antibody labeling in neonatal/adult rats and mice, including in genetically specified excitatory and inhibitory neurons in mice, established that these channels are present in neurons in the pre-BötC region and adjacent medullary respiratory-related regions as well as in motor nuclei known to contain respiratory neurons. We assayed for TRPM4 and TRPC3 channel mRNA in identified inspiratory pre-BötC neurons and motoneurons, which has previously not been performed, although the presence of these channels has been suggested by TRPM4/5 mRNA or TRPC3/C7 channel protein detection in bulk tissue obtained from the pre-BötC region (Crowder et al., 2007; Ben-Mabrouk and Tryba, 2010). Similarly, TRPM4 channel mRNA has been detected in lasercaptured XII rat motoneurons (Alvares et al., 2014), and immunolabeling of TRPM4 channel protein in mouse NA has been reported (Del Negro et al., 2010). These approaches have not specifically established channel expression in respiratory neurons.

Our scmRT-PCR and immunohistological analyses identified TRPM4 and/or TRPC3 mRNA in both excitatory and inhibitory pre-BötC inspiratory neurons in slices from neonatal rats and mice. Coexpression of mRNA for these two channels in single pre-BötC inspiratory neurons was 
A

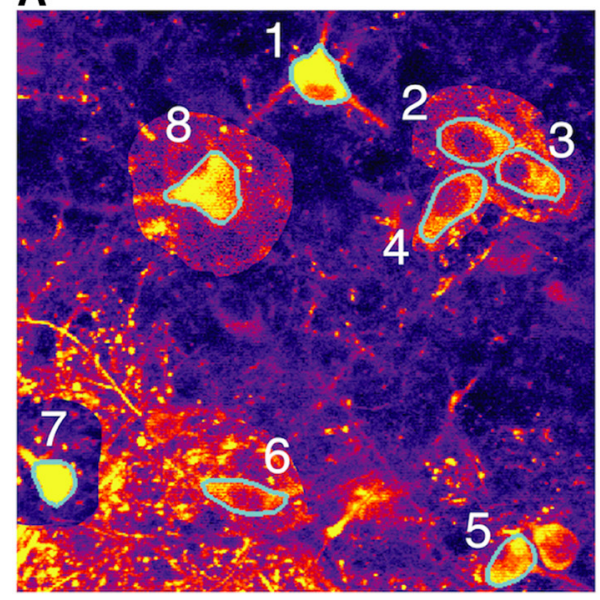

B Control

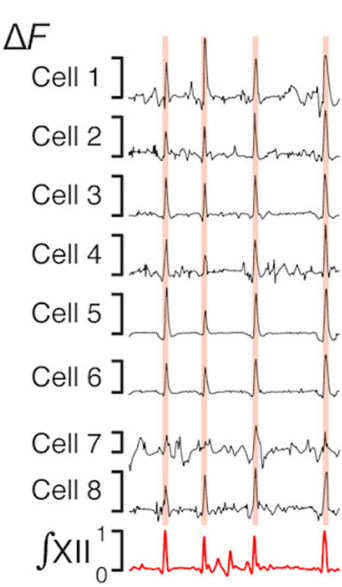

9-Phenanthrol $10 \mathrm{~min}$

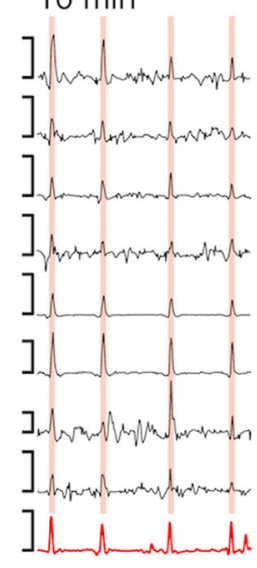

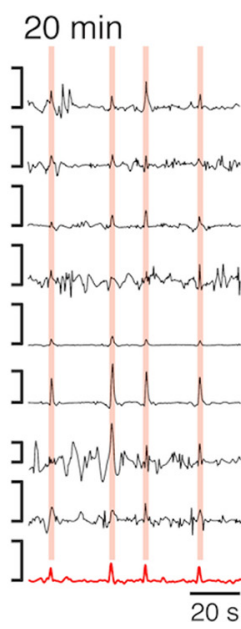

Figure 9. Single-neuron GCaMP6f fluorescence signal tracking during TRPM4 channel inhibition. $\boldsymbol{A}$, Single optical plane image of the pre-BötC region with cells of interest (1-8) in a rhythmically active neonatal medullary in vitro slice preparation from the VgluT2GCaMP6f transgenic mouse. Regions of interest detected algorithmically (see Methods) for quantifying somal fluorescence transients are outlined in cyan. Color scale of pixels immediately surrounding some of the cells $(2,3,7,8)$ was adjusted to more clearly delineate neuron soma in this image. $\boldsymbol{B}$, Examples of time series of single-neuron GCaMPF6f fluorescence transients synchronous with integrated inspiratory hypoglossal activity $\left(\int \mathrm{XII}\right.$, red) used for single-neuron $\Delta F$ analysis during control period, and 10 and 20 min after bath application of $50 \mu \mathrm{m}$ 9-phenanthrol.

found in nearly half of the excitatory neurons. Our sample of inspiratory pre-BötC inhibitory neurons was not sufficient to allow conclusions about channel coexpression in these neurons. Although TRPM5 mRNA (Crowder et al., 2007) and TRPC7 channel protein (Ben-Mabrouk and Tryba, 2010) in the pre-BötC region has been reported, we did not systematically probe for other TRPM/C channels in our sample of pre-BötC inspiratory neurons. Consistent with our immunolabeling results, we also found mRNA for TRPM4 and TRPC3 channels in functionally identified XII and NA inspiratory motoneurons. In general, our findings imply that TRPM4/C3 channels may be func-
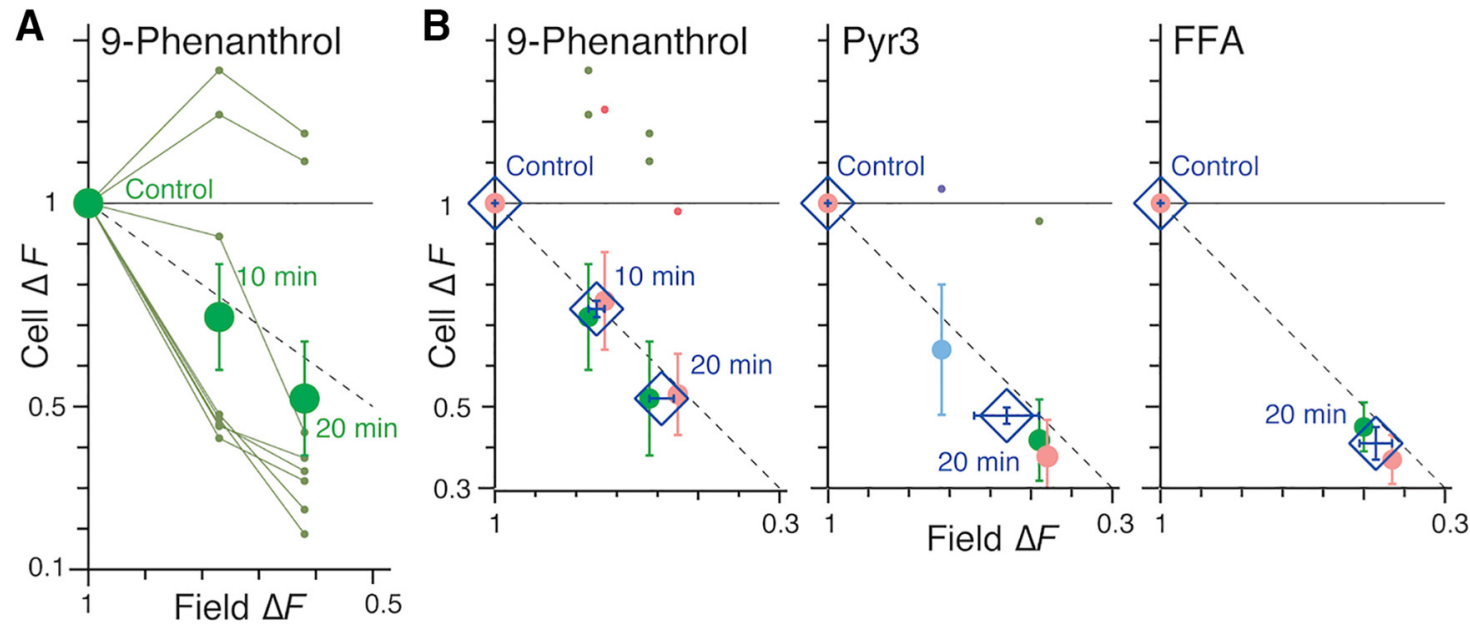

Figure 10. Effects of TRPM4, TRPC3, and $I_{\text {CAN }}$ channel inhibitors on inspiratory $\mathrm{Ca}^{2+}$ activity of the pre-BötC field and glutamatergic neurons expressing GCaMP6f. $\boldsymbol{A}$, Example of relationship between normalized pre-BötC peak field GCaMP6f $\Delta \mathrm{F}$ and normalized individual inspiratory cell $\Delta \mathrm{F}, 10$ and $20 \mathrm{~min}$ after bath-applied $50 \mu \mathrm{M}$ 9-phenanthrol. Eight neurons (shown in Fig. 9) were tracked through time (connected green dots) within the two-photon optical section using automated ROI detection. Group mean values \pm SEM of the normalized fluorescence transients are plotted (green filled circles with error bars) and the identity line (dashed) is indicated. Note that two of the neurons showed augmented fluorescence transients in this example, but the mean group cellular $\Delta F$ nevertheless followed the field $\Delta F$. B. Group summary of effects of TRPM4, TRPC3, and $I_{\text {CAN }}$ inhibitors on the inspiratory pre-BötC field $\Delta F$ and cellular $\Delta F$. Left: mean values of cellular $\Delta F$ (red, $n=6$ neurons; green, $n=8$, same as $\boldsymbol{A}$ ) \pm SEM during control period, $10 \mathrm{~min}$, and $20 \mathrm{~min}$ after bath-applied 9-phenanthrol from two slices are indicated (diamonds and error crosses). Inspiratory neurons with unaffected or increased $\Delta \mathrm{F}$ amplitude included in the group statistics are plotted individually at the top. Middle: three-experiment summary for Pyr3 (red, $n=6$ neurons; green, $n=13$; blue, $n=4$ ). Right: two-experiment summary for FFA (red, $n=12$ neurons; green, $n=6$ ). Identity line (dashed) is indicated. 
A

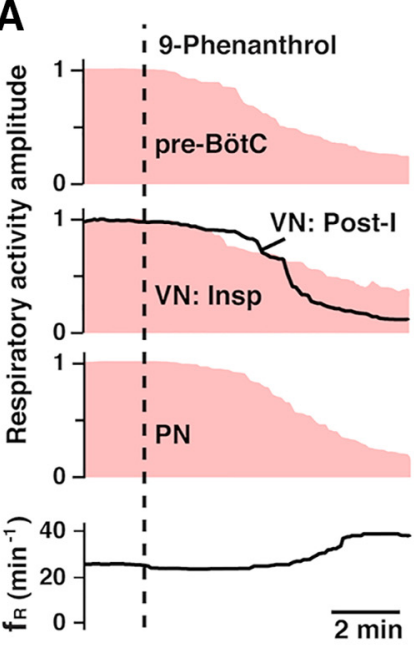

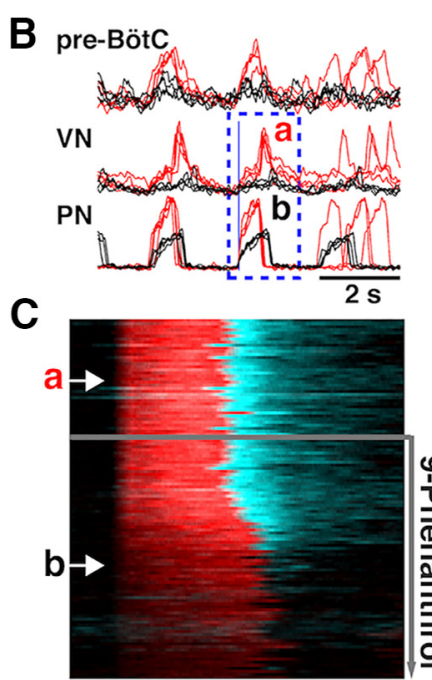

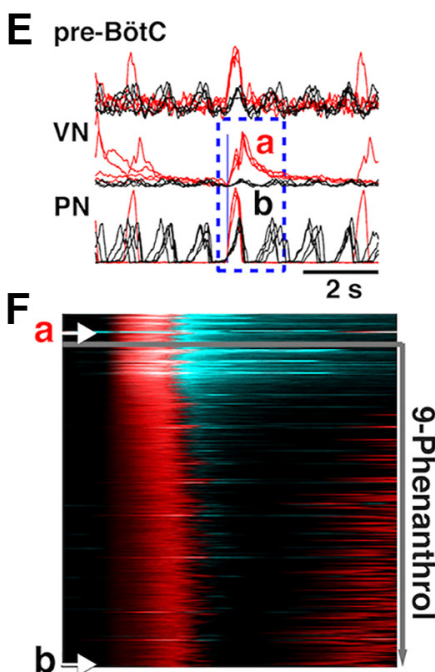

Figure 11. Time courses of perturbations of respiratory neural activities by TRPM4 channel inhibitor in mature rat and mouse arterially perfused in situ brainstem-spinal cord preparations. A-C. Time courses of integrated burst amplitudes (normalized to mean control amplitudes, pink mountain plots) of inspiratory pre-BötC neural population activity (pre-BötC) obtained by extracellular recordings, vagus nerve (VN) inspiratory (Insp) and postinspiratory (post-I) activity (solid black line in middle panel in $\boldsymbol{A}$ ), phrenic nerve (PN) inspiratory activity, and respiratory frequency $\left(f_{\mathrm{R}}\right)$ in perfused preparation from mature (4-wk-old) rat. TRPM4 inhibitor 9-phenanthrol $(50 \mu \mathrm{M})$ was added to the perfusate at the vertical dashed line. B, Cycle-triggered overplots of the three neurograms (pre-BötC, VN, and PN) digitally triggered at the onset of PN activity (vertical solid line) before (red, corresponding to time points marked by arrow a in $\boldsymbol{C}$ ) and after the inhibitor (black traces, at arrow b in $\boldsymbol{C}$ ). $\boldsymbol{C}$, Dynamic raster plots of cycle-triggered PN inspiratory (red) and VN including post-I (cyan, right side) activities. After 9-phenanthrol, $T_{1}$ (red) was prolonged, PN and VN inspiratory amplitude declined (darkened red), and $f_{\mathrm{R}}$ increased (see $\boldsymbol{A}$ ) as post-I activity amplitude declined. $\boldsymbol{D}-\boldsymbol{F}$, Same type of data sets and analysis for an adult (4-mo-old) mouse preparation showing perturbations of pre-BötC, VN, and PN activity, including loss of VN post-I activity and associated increase of $f_{\mathrm{R}}$, following administration of 9-phenanthrol $(20 \mu \mathrm{M})$.

tionally involved at multiple levels within medullary respiratory circuits.

\section{Role of TRPM4 and TRPC3 channels in respiratory pattern generation in vitro}

We analyzed functional contributions of TRPM4 and TRPC3 channels with the selective pharmacological inhibitors 9-phenanthrol and Pyr3, respectively, initially within rhythmically active in vitro slices from neonatal rats and mice. Perturbations of circuit activity were compared with those caused by FFA, a blocker of $I_{\text {CAN }}$ and TRPM4 (Guinamard et al., 2013), that has been used previously to evaluate roles of this current in generating rhythmic bursting activity of respiratory neurons and circuits in vitro (e.g., Peña et al., 2004; Del Negro et al., 2005). We empirically determined from initial in vitro studies and subsequently used concentrations (maximally $50 \mu \mathrm{M}$ for the data presented) of 9-phenanthrol, Pyr3, and FFA that produced large or near-maximal perturbations of the amplitude of inspiratory motor outputs in vitro, and are also expected to be effective and relatively selective for TRPM4, TRPC3, and $I_{\text {CAN }}$ in physiologic preparations (Kiyonaka et al., 2009; Guinamard et al., 2013, 2014). The three channel inhibitors similarly reduced the amplitude of XII inspiratory motoneuronal activity in vitro without significant perturbations of inspiratory frequency and regularity of the rhythm. These amplitude perturbations indicate that currents inhibited by 9-phenanthrol, Pyr3, or FFA are endogenously active in respiratory neurons in vitro. The similar perturbations with 9-phenanthrol and FFA are consistent with the proposal from pharmacological studies in other cells and tissues that FFA blocks TRPM4 channels (Guinamard et al., 2014), as well as the proposal that TRPM4, known to be directly activated by increases in intracellular $\mathrm{Ca}^{2+}$, mediates an I CAN (Launay et al., 2002; Hofmann et al., 2003) active in respiratory neurons.

In previous pharmacological studies investigating the role of $I_{\text {CAN }}$ in active respiratory circuits in neonatal mouse slices in vitro (Peña et al., 2004), high concentrations of FFA $(500 \mu \mathrm{M})$ reduced the amplitude/area of integrated inspiratory population activity but caused a relatively small $(\sim 20 \%)$ reduction of inspiratory burst frequency without affecting regularity of the rhythm. Although these high concentrations of FFA can also depress voltagegated $\mathrm{Na}^{+}$currents (at $>100 \mu \mathrm{M}$ in hippocampal neurons; Yau et al., 2010) and voltage-gated $\mathrm{Ca}^{2+}$ currents (Shimamura et al., 2002), as well as cause other nonselective perturbations of neuronal excitability (Guinamard et al., 2013), these observations are generally consistent with our results with FFA in both neonatal rat and mouse in vitro slices showing relatively large perturbations of the amplitude of pre-BötC and XII motoneuronal inspiratory activity and only small perturbations of inspiratory frequency without affecting stability of the inspiratory rhythm. In other earlier in vitro mouse slice experiments, $100 \mu \mathrm{M}$ FFA reduced inspiratory drive potentials of individual pre-BötC neurons, but without reducing the amplitude or altering the frequency of XII motor discharge (Del Negro et al., 2005; Pace et al., 2007). The lack of perturbations of XII inspiratory activity at this concentration of 
A

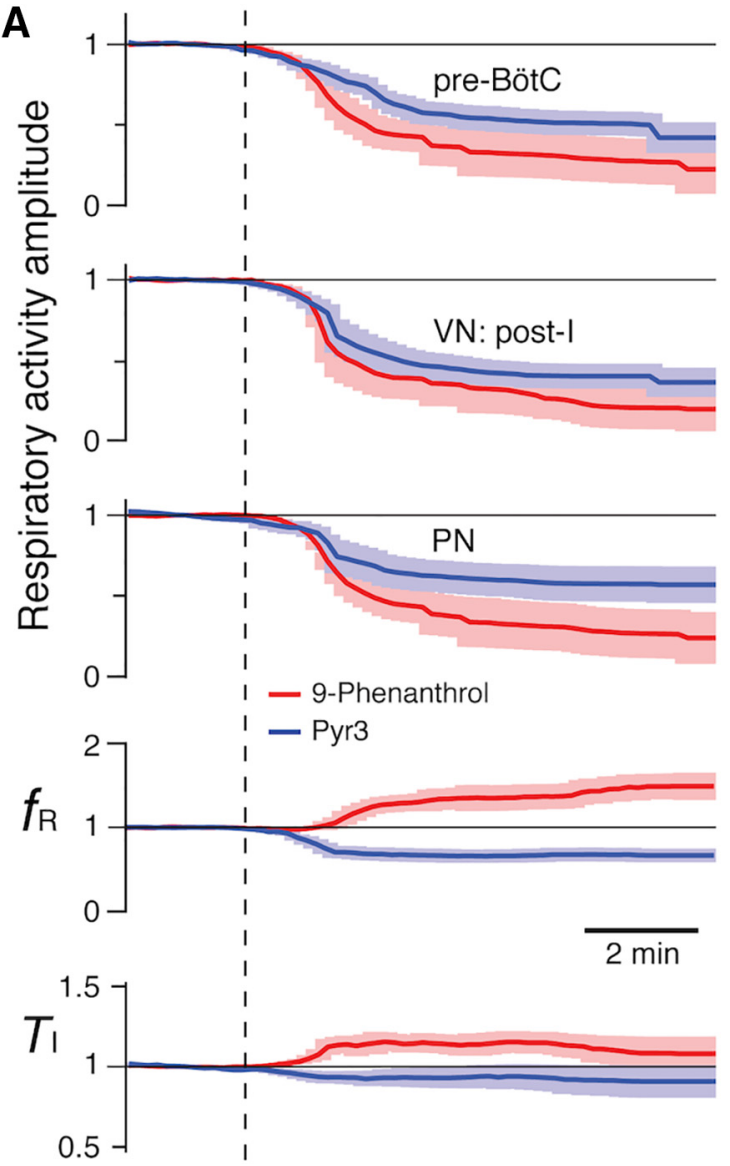

B

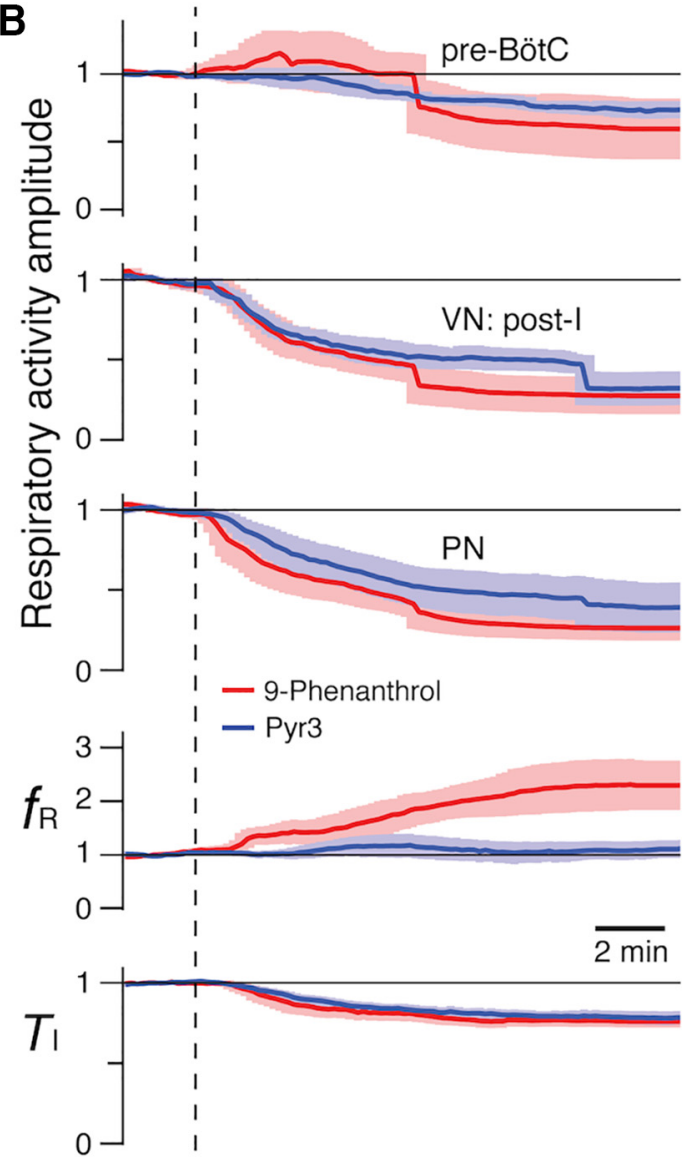

Figure 12. Summary of effects of TRPM4 and TRPC3 channel inhibitors on respiratory activities in arterially perfused brainstem-spinal cord preparation in situ from mature rats and adult mice. $\boldsymbol{A}, \boldsymbol{B}$, Summary time courses (solid lines: mean values; lighter color bands: \pm SEM) of the amplitudes of integrated inspiratory pre-BötC neural population activity, VN post-inspiratory (post-I) activity, and PN inspiratory activity from rat $(\boldsymbol{A}, 9$-phenanthrol, $n=6$; Pyr3, $n=8)$ and mouse (B, 9-phenanthrol, $n=7$; Pyr3, $n=6)$ preparations, showing significant reduction of all amplitudes (normalized to mean control values) by both TRPM4 (9-phenanthrol, red) and TRPC3 (Pyr3, blue) channel inhibitors. Bottom panels show group summaries for inspiratory frequency $\left(f_{\mathrm{R}}\right)$ and inspiratory activity time $\left(T_{1}\right)$. Time of drug administration (9-phenanthrol, 20-50 $\mu \mathrm{m}$; Pyr3, $50 \mu \mathrm{M}$ ) is indicated by vertical dashed lines.

FFA is difficult to reconcile with our electrophysiological and $\mathrm{Ca}^{2+}$ imaging results showing correlations between the amplitude of pre-BötC inspiratory activity, as assessed by intracellular $\mathrm{Ca}^{2+}$ dynamics (below), and the reduction of XII inspiratory discharge amplitude. In those earlier studies, however, higher concentrations (300-350 $\mu \mathrm{M})$ of FFA that are considered nonselective for $I_{\text {CAN }}$ reduced the XII inspiratory activity amplitude and could have eliminated rhythmic inspiratory XII motor output.

Consistent with our ScmRT-PCR results indicating TRPC3 channel mRNA expression in identified inspiratory neurons, the amplitude perturbations produced by Pyr3 suggest that TRPC 3 channels are also functionally activated during respiratory circuit activity in vitro. These channels are not directly $\mathrm{Ca}^{2+}$ activated, but mediate $\mathrm{Na}^{+} / \mathrm{Ca}^{2+}$ currents, and may be involved in regulating neuronal $\mathrm{Ca}^{2+}$-related signaling (Talavera et al., 2008; Birnbaumer, 2009; Guinamard et al., 2013) in respiratory neurons. This TRPC3 channel-mediated $\mathrm{Ca}^{2+}$ flux/intracellular $\mathrm{Ca}^{2+}$ regulation can potentially also affect TRPM4/I CAN in respiratory neurons. We found coexpression of mRNA for TRPC3 and TRPM4 channels in approximately half of the excitatory
pre-BötC inspiratory neurons and all of the respiratory motoneurons assayed, suggesting that such a functional interaction may be possible. The roles of TRPC3-mediated cationic currents $/ \mathrm{Ca}^{2+}$-related signaling in generating respiratory neuron activity have not been previously investigated. Our results suggest an important functional role of these channels in activity amplitude modulation but not in rhythmogenesis, like TRPM $4 / I_{\text {CAN }}$ channels. Whether the similar amplitude perturbations by the TRPC3 and TRPM4 channel inhibitors reflects involvement with TRPM4/ $I_{\text {CAN }}$ activation by $\mathrm{Ca}^{2+}$-related functions of TRPC3 channels remains to be determined.

In general, the optimal pharmacological strategy for probing the roles of TRPM4/I CAN $_{\text {ON }}$ ORPC3 channels active in respiratory circuit neurons has not been definitively established. The major problem is to identify and analyze the neuronal currents attenuated by the channel inhibitors in respiratory neurons at any applied concentrations. Measurements of whole-cell currents mediated by TRPM $4 / I_{\text {CAN }}$ or TRPC3 channels in respiratory neurons have not yet been performed, so that 9-phenanthrol and Pyr3 concentrations likely to be effective/selective have been inferred 
in part from pharmacological analyses performed in other (typically nonneuronal) cell types (Kiyonaka et al., 2009; Guinamard et al., 2014). The problem is particularly complicated for resolving the $\mathrm{Ca}^{2+}$-activated TRPM4/I $\mathrm{CAN}^{-}$ mediated currents, because the sources of $\mathrm{Ca}^{2+}$ flux activating these currents in respiratory neurons need to be preserved and taken into account in a detailed pharmacological analysis of currents activated endogenously during respiratory neuronal activity. Although $\mathrm{Ca}^{2+}$ flux through voltage-gated $\mathrm{Ca}^{2+}$ channels (Peña et al., 2004; Morgado-Valle et al., 2008), and/or synaptically activated $\mathrm{Ca}^{2+}$-fluxes, including through ionotropic glutamatergic receptors and/or activation of metabotropic glutamatergic receptors to induce $\mathrm{ER} \mathrm{Ca}^{2+}$ release, have been postulated to activate $I_{\text {CAN }}$ (Pace et al., 2007; Mironov, 2008; Del Negro et al., 2010), these mechanisms have not been established.

\section{Correlated perturbations of pre-BötC excitatory neuronal population activity and hypoglossal motor output in vitro}

We initially evaluated roles of TRPM4 and TRPC 3 channels by analyzing perturbations of XII inspiratory motor output in slices, but this approach does not necessary allow assessment of the contributions of these channels in pre-BötC neurons to perturbations of the motor output, since TRPM4 and TRPC3 channels are also expressed in XII inspiratory neurons. We also note that TRPM4 or TRPC3 channel antibody labeling was identified in regions of the reticular formation dorsal to the pre-BötC region that contains inspiratory XII premotoneurons (Koizumi et al., 2008; Revill et al., 2015). Accordingly, reductions in XII activity amplitude by channel inhibitors in our in vitro slice preparations potentially reflect reduced activity of XII motoneurons and possibly other neurons within inspiratory drive transmission circuits. We therefore more directly established involvement of pre-BötC neurons by dynamic $\mathrm{Ca}^{2+}$ imaging of inspiratory pre-BötC neuronal activity in slices from transgenic mice expressing GCaMP6f in glutamatergic neurons during bath application of the channel inhibitors. This allowed us to assess activity perturbations of the critical populations of pre-BötC excitatory neurons generating inspiratory rhythm and synaptic drive in transmission circuits to XII motoneurons, and to compare simultaneous perturbations of activity of these neurons and XII motor output. All of the channel inhibitors caused reductions in the amplitude of spatially averaged GCaMP6 $f$ fluorescence transients (field $\Delta F$ ) and the fluorescence transients of individual glutamatergic inspiratory pre-BötC neurons (cell $\Delta F$ ), confirming inspiratory activity perturbations at the level of pre-BötC excitatory neurons. Furthermore, we established that the field $\Delta F$ reflects the mean cell $\Delta F$ of sets of individual excitatory inspiratory neurons. We also found significantly correlated, linear relationships (Fig. 8) between the amplitude of field $\Delta F$ and the amplitude of integrated XII inspiratory activity. The slopes of these linear relationships for 9-phenanthrol, FFA, and Pyr3 $(0.86,0.68$, and 0.46 , respectively), particularly with Pyr3, reflect that initially the activity amplitude perturbations of our imaged sample of inspiratory glutamatergic neurons tended to occur more rapidly than the reduction in integrated XII activity, but the group mean amplitude perturbations tended to converge toward the identity line of the field $\Delta F$ versus integrated XII amplitude relationships as the quasi-steady-state perturbations were reached. Our results suggest that the number of active pre-BötC inspiratory cells and the burst amplitude of each active pre-BötC neuron after application of the channel inhibitors are important factors contributing to the overall reduction of pre-BötC field $\Delta F$ and decrease of XII burst amplitude.

We conclude that the perturbations of pre-BötC inspiratory activity correlate with the perturbation of XII inspiratory activity amplitude. The $\Delta F$ amplitude perturbations occurred without significant changes in the frequency of inspiratory-related activity within the pre-BötC and simultaneously recorded XII inspiratory activity. The extent to which activity perturbations of XII inspiratory motoneurons or neurons within the rhythmic inspiratory drive transmission premotor circuits contribute to the reduction of XII motor output remains to be determined. We also note that there is a subpopulation of pre-BötC inspiratory neurons with axonal projections to XII motoneurons (Koizumi et al., 2008,2013 ), and reduced activity of these neurons may also contribute to the overall reduction of inspiratory activity in the transmission circuits without perturbing rhythm generation.

\section{Contributions of TRPM4 and TRPC3 channels to respiratory pattern generation in mature rodent brainstem circuits in situ}

We also analyzed functional contributions of TRPM4 and TRPC3 channels with 9-phenanthrol and Pyr3, respectively, in mature rat and mouse arterially perfused in situ brainstem-spinal cord preparations to evaluate roles of these channels in more intact circuits generating a eupneic-like three-phase respiratory motor output pattern. Moreover, extending our analysis to mature animals was necessary, since $I_{\text {CAN }}$-dependent neuronal bursting, and accordingly potential involvement of TRPM4, has been proposed to contribute to inspiratory rhythm generation predominantly in mice older than P5 (Peña et al., 2004; Del Negro et al., 2005), although $I_{\text {CAN }}$ is postulated to contribute to formation of drive potentials generating inspiratory bursts throughout development (Del Negro et al., 2005). In agreement with our results obtained in vitro, presumptive inhibition of TRPM4 or TRPC3 channels in the more intact rat and mouse circuits in situ significantly reduced the amplitude of pre-BötC inspiratory activity, accompanied by reduced amplitudes of inspiratory motor outputs as evaluated from integrated vagal and phrenic nerve inspiratory activities. In addition, the channel inhibitors, especially 9-phenanthrol, caused large reductions in vagal post-I activity (e.g., Fig. 11), indicating an important contribution of endogenous channel activation to inspiratory-expiratory pattern generation in more intact respiratory circuits. The increase of respiratory frequency, primarily with TRPM4 channel inhibition, occurred with the reduction of expiratory phase duration as post-I activity was reduced (Smith et al., 2007), although rhythm generation was not disrupted. 
The reduction of post-I vagal activity could reflect contributions of TRPM4 and TRPC 3 channel activation at the level of vagal motoneurons, or at the interneuronal level in excitatory/inhibitory circuits in ventral medullary respiratory-related regions, including within the BötC, that generate post-I activity (Smith et al., 2007; Richter and Smith, 2014) and where these channels may be present in excitatory/inhibitory neurons as suggested by our immunolabeling results. According to the respiratory central pattern generation (CPG) network model based on experimental analyses with in situ preparations (Rubin et al., 2009b), different types (e.g., neurotransmitter phenotypes, active phase, bursting pattern) of respiratory interneurons in the pre-BötC and BötC are functionally interacting to generate a normal three-phase pattern of respiratory neural activity. Our experimental results of different effects of TRPM4 or TRPC3 channel inhibition on $f_{\mathrm{R}}$ in the more intact in situ preparations (Fig. 12) suggest different sensitivity to TRPM4 or TRPC3 channel inhibitors among different types of respiratory neurons in the CPG circuits. Contributions of TRPM4 and TRPC 3 channel activation in different types of excitatory and/or inhibitory respiratory neurons remain to be clarified.

Our results suggest that inhibiting TRPM $4 / /_{\text {CAN }}$ or TRPC3 in excitatory pre-BötC inspiratory neurons primarily contributes to the amplitude decrease of inspiratory motor outputs in vitro and in situ, whereas inhibiting TRPM4 or TRPC3 channels in inhibitory neurons, possibly BötC expiratory neurons, in the more intact in situ circuits causes perturbations of post-I activity (e.g., Marchenko et al., 2016) and therefore respiratory frequency. In summary, we suggest that endogenous activation of TRPM4/ $I_{\text {CAN }}$ or TRPC3 plays an important role in regulating activity of excitatory and inhibitory respiratory neurons, the latter particularly in the intact in situ CPG circuits for inspiratory-expiratory pattern generation.

\section{Implications for proposed $I_{\mathrm{CAN}}$-dependent and other mechanisms of respiratory rhythm generation in pre- BötC circuits}

Based on previous experimental studies in neonatal mouse rhythmic medullary slices in vitro (Crowder et al., 2007; Pace et al., 2007; Pace and Del Negro, 2008) and organotypic pre-BötC cultures (Mironov, 2008, 2013), and also computational modeling studies (Rubin et al., 2009a; Dunmyre et al., 2011), an emergent $I_{\mathrm{CAN}}$-dependent mechanism in pre-BötC excitatory circuits was postulated to play a major role in respiratory rhythmogenesis according to the "group pacemaker" hypothesis (Feldman and Del Negro, 2006; Del Negro et al., 2010). In this model, synapticallyactivated $\mathrm{Ca}^{2+}$ fluxes, especially mediated by metabotropic glutamate receptors (mGluRs), were proposed to trigger $I_{\text {CAN }}$ activation through intracellular $\mathrm{Ca}^{2+}$ signaling involving inositol triphosphate $\left(\mathrm{IP}_{3}\right)$-mediated $\mathrm{Ca}^{2+}$ release from $\mathrm{ER}$ stores. Activation of $I_{\text {CAN }}$ is proposed to generate depolarization of excitatory inspiratory pre-BötC neurons to primarily produce synaptically mediated (i.e., network-dependent) inspiratory drive potentials underlying inspiratory bursts (Crowder et al., 2007; Pace et al., 2007; Pace and Del Negro, 2008). During population-level inspiratory bursts, the $I_{\mathrm{CAN}^{-}}$ dependent depolarization has been suggested to cause partial voltage-dependent inactivation of neuronal spikegenerating transient $\mathrm{Na}^{+}$channels, associated with transient depression of recurrent excitation and circuit-generated excitatory synaptic drive to deactivate $I_{\mathrm{CAN}}$ and terminate inspiratory bursts (Rubin et al., 2009a; Del Negro et al., 2010). In other more complex models with multiple sources of neuronal $\mathrm{Ca}^{2+}$ flux (Jasinski et al., 2013; Rybak et al., 2014), including voltage-gated $\mathrm{Ca}^{2+}$ currents, it has been theoretically shown that $I_{\mathrm{CAN}}$-induced bursting, and subsequent burst termination sufficient for rhythmogenesis, can occur by dynamic $\mathrm{Ca}^{2+}$-dependent activation-inactivation of $\mathrm{IP}_{3}$ receptor-mediated $\mathrm{Ca}^{2+}$ release, without or with involvement of other burst-terminating mechanisms such as $\mathrm{Na}^{+} / \mathrm{K}^{+}$pump currents. The $\mathrm{Na}^{+} / \mathrm{K}^{+}$pump currents can hypothetically contribute importantly to inspiratory burst termination and may be regulated by $I_{\mathrm{CAN}}-$ mediated $\mathrm{Na}^{+}$flux, linking $I_{\text {CAN }}$ activation to another mechanism for inspiratory burst termination critical for rhythm generation. Interfering with ER $\mathrm{Ca}^{2+}$ release mechanisms does not disturb inspiratory rhythm generation in the pre-BötC in vitro, however, indicating that normally this source of $\mathrm{Ca}^{2+}$ flux is not critically involved in rhythm generation or control of inspiratory amplitude in vitro (Beltran-Parrazal et al., 2012), so other $\mathrm{Ca}^{2+}$ sources explored in these models seem to be involved in activating $I_{\text {CAN. }}$.

Another important hypothesis for inspiratory rhythm generation long proposed in the field is that $I_{\text {CAN }}$-dependent, FFA-sensitive pre-BötC inspiratory pacemaker neurons (i.e., inspiratory neurons with intrinsic oscillatory bursting properties when isolated from synaptic inputs), with $I_{\text {CAN }}$ activation driven by voltage-gated, $\mathrm{Cd}^{+2}$-sensitive $\mathrm{Ca}^{2+}$ currents, have a critical rhythmogenic role (Peña et al., 2004) in pre-BötC circuits together with other populations of neurons with oscillatory bursting properties mediated by persistent $\mathrm{Na}^{+}$current $\left(I_{\mathrm{NaP}}\right.$; dual pacemaker hypothesis; Thoby-Brisson and Ramirez, 2001; Peña et al., 2004; Ramirez et al., 2011), following the proposal of $I_{\mathrm{NaP}}$-dependent cellular and excitatory population rhythm generation mechanisms in pre-BötC excitatory circuits (Butera et al., 1999a, b).

In general, our results do not support the concept that populations of pre-BötC neurons with $I_{\text {CAN }}$-mediated bursting properties are critically involved in generating inspiratory rhythm. However, they support the proposal that TPRM $4 / I_{\mathrm{CAN}}$-mediated currents are functionally active in respiratory neurons and importantly contribute to inspiratory burst generation determining the amplitude of pre-BötC neuronal population activity. TRPC3 channels also have this fundamental role, possibly by providing $\mathrm{Ca}^{2+}$ flux activating TRPM4// CAN. Remarkably, this amplitude control is essentially independent of the inspiratory rhythm generation mechanism and indicates there is a rhythmogenic kernel subpopulation of neurons within the pre-BötC excitatory network that rely on a fundamentally different oscillatory mechanism. Previous studies have proposed (Butera et al., 1999a, b) and presented evidence (Koizumi and Smith, 2008) that $I_{\mathrm{NaP}}$-dependent mechanisms are sufficient to account for a number of features of inspiratory rhythm generation when neonatal pre-BötC 
circuits are isolated in vitro, as well as in reduced in situ preparations from mature rats (Smith et al., 2007). In the more intact mature system, this $I_{\mathrm{NaP}}$-dependent oscillatory mechanism may not be sufficient to explain rhythm generation, which involves more complex sets of inhibitory circuit interactions with the pre-BötC excitatory circuits (Smith et al., 2007; Rubin et al., 2009b). The present studies indicate that although TPRM $4 / I_{\text {CAN }}$-mediated currents are functionally active in the more intact system and have a basic role in inspiratory-expiratory respiratory pattern generation, they are also not essential for rhythm generation.

\section{References}

Alvares TS, Revill AL, Huxtable AG, Lorenz CD, Funk GD (2014) P2Y1 receptor-mediated potentiation of inspiratory motor output in neonatal rat in vitro. J Physiol 592:3089-3111. CrossRef Medline

Beltran-Parrazal L, Fernandez-Ruiz J, Toledo R, Manzo J, MorgadoValle C (2012) Inhibition of endoplasmic reticulum $\mathrm{Ca}^{2+}$ ATPase in preBötzinger complex of neonatal rat does not affect respiratory rhythm generation. Neuroscience 224:116-124. CrossRef

Ben-Mabrouk F, Tryba AK (2010) Substance P modulation of TRPC3/7 channels improves respiratory rhythm regularity and ICAN-dependent pacemaker activity. Eur J Neurosci 31:12191232. CrossRef Medline

Birnbaumer L (2009) The TRPC class of ion channels: a critical review of their roles in slow, sustained increases in intracellular $\mathrm{Ca}^{2+}$ concentrations. Annu Rev Pharmacol Toxicol 49:395-426. CrossRef Medline

Brayden JE, Earley S, Nelson MT, Reading S (2008) Transient receptor potential (TRP) channels, vascular tone and autoregulation of cerebral blood flow. Clin Exp Pharmacol Physiol 35:1116-1120. CrossRef Medline

Butera RJ, Jr., Rinzel J, Smith JC (1999a) Models of respiratory rhythm generation in the pre-Bötzinger complex. I. Bursting pacemaker neurons. J Neurophysiol 82:382-397.

Butera RJ, Jr., Rinzel J, Smith JC (1999b) Models of respiratory rhythm generation in the pre-Bötzinger complex. II. Populations Of coupled pacemaker neurons. J Neurophysiol 82:398-415.

Chen Y, Song X, Ye S, Miao L, Zhu Y, Zhang RG, Ji G (2013) Structural insight into enhanced calcium indicator GCaMP3 and GCaMPJ to promote further improvement. Protein Cell 4:299-309. CrossRef Medline

Crowder EA, Saha MS, Pace RW, Zhang H, Prestwich GD, Del Negro CA (2007) Phosphatidylinositol 4,5-bisphosphate regulates inspiratory burst activity in the neonatal mouse preBötzinger complex. J Physiol 582:1047-1058. CrossRef Medline

Del Negro CA, Hayes JA, Pace RW, Brush BR, Teruyama R, Feldman JL (2010) Synaptically activated burst-generating conductances may underlie a group-pacemaker mechanism for respiratory rhythm generation in mammals. Prog Brain Res 187:111-136. CrossRef Medline

Del Negro CA, Morgado-Valle C, Hayes JA, Mackay DD, Pace RW, Crowder EA, Feldman JL (2005) Sodium and calcium currentmediated pacemaker neurons and respiratory rhythm generation. J Neurosci 25:446-453. CrossRef Medline

Dunmyre JR, Del Negro CA, Rubin JE (2011) Interactions of persistent sodium and calcium-activated nonspecific cationic currents yield dynamically distinct bursting regimes in a model of respiratory neurons. J Comput Neurosci 31:305-328. CrossRef

Feldman JL, Del Negro CA (2006) Looking for inspiration: new perspectives on respiratory rhythm. Nat Rev Neurosci 7:232-242. CrossRef Medline

Feng S, Li H, Tai Y, Huang J, Su Y, Abramowitz J, Zhu MX, Birnbaumer L, Wang $Y$ (2013) Canonical transient receptor potential 3 channels regulate mitochondrial calcium uptake. Proc Natl Acad Sci U S A 110:11011-11016. CrossRef Medline
Fishman M, Jacono FJ, Park S, Jamasebi R, Thungtong A, Loparo KA, Dick TE (2012) A method for analyzing temporal patterns of variability of a time series from Poincaré plots. J Appl Physiol (1985) 113:297-306. CrossRef

Gong S, Doughty M, Harbaugh CR, Cummins A, Hatten ME, Heintz N, Gerfen CR (2007) Targeting Cre recombinase to specific neuron populations with bacterial artificial chromosome constructs. J Neurosci 27:9817-9823. CrossRef Medline

Gray PA, Janczewski WA, Mellen N, McCrimmon DR, Feldman JL (2001) Normal breathing requires preBötzinger complex neurokinin-1 receptor-expressing neurons. Nat Neurosci 4:927930. CrossRef Medline

Guinamard R, Demion M, Launay P (2010) Physiological roles of the TRPM4 channel extracted from background currents. Physiology (Bethesda) 25:155-164. CrossRef Medline

Guinamard R, Simard C, Del Negro C (2013) Flufenamic acid as an ion channel modulator. Pharmacol Ther 138:272-284. CrossRef Medline

Guinamard R, Hof T, Del Negro CA (2014) The TRPM4 channel inhibitor 9-phenanthrol. Br J Pharmacol 171:1600-1613. CrossRef Medline

Hofmann T, Chubanov V, Gudermann T, Montell C (2003) TRPM5 is a voltage-modulated and $\mathrm{Ca}^{2+}$-activated monovalent selective cation channel. Curr Biol 13:1153-1158. CrossRef

Jasinski PE, Molkov YI, Shevtsova NA, Smith JC, Rybak IA (2013) Sodium and calcium mechanisms of rhythmic bursting in excitatory neural networks of the pre-Bötzinger complex: a computational modelling study. Eur J Neurosci 37:212-230. CrossRef Medline

Kiyonaka S, et al. (2009) Selective and direct inhibition of TRPC3 channels underlies biological activities of a pyrazole compound. Proc Natl Acad Sci U S A 106:5400-5405. CrossRef Medline

Koizumi H, Smith JC (2008) Persistent $\mathrm{Na}^{+}$and $\mathrm{K}^{+}$-dominated leak currents contribute to respiratory rhythm generation in the preBötzinger complex in vitro. J Neurosci 28:1773-1785. CrossRef

Koizumi H, Wilson CG, Wong S, Yamanishi T, Koshiya N, Smith JC (2008) Functional imaging, spatial reconstruction, and biophysical analysis of a respiratory motor circuit isolated in vitro. $\mathrm{J}$ Neurosci 28:2353-2365. CrossRef Medline

Koizumi H, Koshiya N, Chia JX, Cao F, Nugent J, Zhang R, Smith JC (2013) Structural-functional properties of identified excitatory and inhibitory interneurons within pre-Bötzinger complex respiratory microcircuits. J Neurosci 33:2994-3009. CrossRef Medline

Koizumi H, Mosher B, Tariq MF, Zhang R, Koshiya N, Smith JC (2016) Voltage-dependent rhythmogenic property of respiratory pre-Bötzinger complex glutamatergic, Dbx1-derived, and somatostatin-expressing neuron populations revealed by graded optogenetic inhibition. eNeuro 3:0081-16. CrossRef

Koshiya N, Smith JC (1999) Neuronal pacemaker for breathing visualized in vitro. Nature 400:360-363. CrossRef Medline

Launay P, Fleig A, Perraud AL, Scharenberg AM, Penner R, Kinet JP (2002) TRPM4 is a $\mathrm{Ca}^{2+}$-activated nonselective cation channel mediating cell membrane depolarization. Cell 109:397-407. Medline

Marchenko V, Koizumi H, Mosher B, Koshiya N, Tariq MF, Bezdudnaya TG, Zhang R, Molkov YI, Rybak IA, Smith JC (2016) Perturbations of respiratory rhythm and pattern by disrupting synaptic inhibition within pre-Bötzinger and Bötzinger complexes. eNeuro 3:0011-16. CrossRef

Mironov SL (2008) Metabotropic glutamate receptors activate dendritic calcium waves and TRPM channels which drive rhythmic respiratory patterns in mice. J Physiol 586:2277-2291. CrossRef Medline

Mironov S (2009) Respiratory circuits: function, mechanisms, topology, and pathology. Neuroscientist 15:194-208. CrossRef Medline

Mironov SL (2013) Calmodulin and calmodulin kinase II mediate emergent bursting activity in the brainstem respiratory network (preBötzinger complex). J Physiol 591:1613-1630. CrossRef Medline 
Morgado-Valle C, Baca SM, Feldman JL (2010) Glycinergic pacemaker neurons in preBötzinger complex of neonatal mouse. $J$ Neurosci 30:3634-3639. CrossRef Medline

Morgado-Valle C, Beltran-Parrazal L, DiFranco M, Vergara JL, Feldman JL (2008) Somatic $\mathrm{Ca}^{2+}$ transients do not contribute to inspiratory drive in preBötzinger Complex neurons. J Physiol 586: 4531-4540. CrossRef

Onimaru H, Homma I (2003) A novel functional neuron group for respiratory rhythm generation in the ventral medulla. $J$ Neurosci 23:1478-1486.

Pace RW, Del Negro CA (2008) AMPA and metabotropic glutamate receptors cooperatively generate inspiratory-like depolarization in mouse respiratory neurons in vitro. Eur J Neurosci 28:2434-2442. CrossRef Medline

Pace RW, Mackay DD, Feldman JL, Del Negro CA (2007) Inspiratory bursts in the preBötzinger complex depend on a calcium-activated non-specific cation current linked to glutamate receptors in neonatal mice. J Physiol 582:113-125. CrossRef Medline

Paton JF (1996) A working heart-brainstem preparation of the mouse. J Neurosci Methods 65:63-68. Medline

Peña F, Parkis MA, Tryba AK, Ramirez JM (2004) Differential contribution of pacemaker properties to the generation of respiratory rhythms during normoxia and hypoxia. Neuron 43:105-117. CrossRef Medline

Pickering AE, Paton JF (2006) A decerebrate, artificially-perfused in situ preparation of rat: utility for the study of autonomic and nociceptive processing. J Neurosci Methods 155:260-271. CrossRef

Ramirez JM, Koch H, Garcia AJ, 3rd, Doi A, Zanella S (2011) The role of spiking and bursting pacemakers in the neuronal control of breathing. J Biol Phys 37:241-261. CrossRef Medline

Revill AL, Vann NC, Akins VT, Kottick A, Gray PA, Del Negro CA, Funk GD (2015) Dbx1 precursor cells are a source of inspiratory XII premotoneurons. Elife 4:e12301.

Richter DW, Smith JC (2014) Respiratory rhythm generation in vivo. Physiology (Bethesda) 29:58-71. CrossRef Medline

Rubin JE, Hayes JA, Mendenhall JL, Del Negro CA (2009a) Calciumactivated nonspecific cation current and synaptic depression promote network-dependent burst oscillations. Proc Natl Acad Sci U S A 106:2939-2944.
Rubin JE, Shevtsova NA, Ermentrout GB, Smith JC, Rybak IA (2009b) Multiple rhythmic states in a model of the respiratory central pattern generator. J Neurophysiol 101:2146-2165.

Rybak IA, Molkov YI, Jasinski PE, Shevtsova NA, Smith JC (2014) Rhythmic bursting in the pre-Bötzinger complex: mechanisms and models. Prog Brain Res 209:1-23. CrossRef Medline

Schattling B, Steinbach K, Thies E, Kruse M, Menigoz A, Ufer F, Flockerzi V, Brück W, Pongs $O$, Vennekens R, Kneussel $M$, Freichel M, Merkler D, Friese MA (2012) TRPM4 cation channel mediates axonal and neuronal degeneration in experimental autoimmune encephalomyelitis and multiple sclerosis. Nat Med 18: 1805-1811. CrossRef Medline

Shevtsova NA, Büsselberg D, Molkov YI, Bischoff AM, Smith JC, Richter DW, Rybak IA (2014) Effects of glycinergic inhibition failure on respiratory rhythm and pattern generation. Prog Brain Res 209:25-38. CrossRef Medline

Shimamura K, Zhou M, Ito Y, Kimura S, Zou LB, Sekiguchi F, Kitramura K, Sunano S (2002) Effects of flufenamic acid on smooth muscle of the carotid artery isolated from spontaneously hypertensive rats. J Smooth Muscle Res 38:39-50. Medline

Smith JC, Ellenberger HH, Ballanyi K, Richter DW, Feldman JL (1991) Pre-Bötzinger complex: a brainstem region that may generate respiratory rhythm in mammals. Science 254:726-729. Medline

Smith JC, Abdala AP, Koizumi H, Rybak IA, Paton JF (2007) Spatial and functional architecture of the mammalian brain stem respiratory network: a hierarchy of three oscillatory mechanisms. J Neurophysiol 98:3370-3387. CrossRef Medline

Talavera K, Nilius B, Voets T (2008) Neuronal TRP channels: thermometers, pathfinders and life-savers. Trends Neurosci 31:287295. CrossRef Medline

Teulon J (2000) $\mathrm{Ca}^{2+}$ Activated Nonselective Cation Channels. Berlin: Springer.

Thoby-Brisson M, Ramirez JM (2001) Identification of two types of inspiratory pacemaker neurons in the isolated respiratory neural network of mice. J Neurophysiol 86:104-112. CrossRef Medline

Tulppo MP, Mäkikallio TH, Takala TE, Seppänen T, Huikuri HV (1996) Quantitative beat-to-beat analysis of heart rate dynamics during exercise. Am J Physiol 271:H244-H252. CrossRef Medline

Yau HJ, Baranauskas G, Martina M (2010) Flufenamic acid decreases neuronal excitability through modulation of voltage-gated sodium channel gating. J Physiol 588:3869-3882. CrossRef 